\title{
Micro-Macro relations for flow through random arrays of cylinders
}

\author{
K. Yazdchi ${ }^{*}$, S. Srivastava and S. Luding \\ Multi Scale Mechanics (MSM), Faculty of Engineering Technology, \\ University of Twente, P.O. Box 217, 7500 AE Enschede, The Netherlands
}

\begin{abstract}
The transverse permeability for creeping flow through unidirectional random arrays of fibres with various structures is revisited theoretically and numerically using the finite element method (FEM). The microstructure at various porosities has a strong effect on the transport properties, like permeability, of fibrous materials. We compare different microstructures (due to four random generator algorithms) as well as the effect of boundary conditions, finite size, homogeneity and isotropy of the structure on the macroscopic permeability of the fibrous medium. Permeability data for different minimal distances collapse when their minimal value is subtracted, which yields an empirical macroscopic permeability master function of porosity. Furthermore, as main result, a microstructural model is developed based on the lubrication effect in the narrow channels between neighboring fibres. The numerical experiments suggest a unique, scaling power law relationship between the permeability obtained from fluid flow simulations and the mean value of the shortest Delaunay triangulation edges (constructed using the centers of the fibres), which is identical to the averaged second nearest neighbor fibre distances. This universal lubrication relation, as valid in a wide range of porosities, accounts for the microstructure, e.g. hexagonally ordered or disordered fibrous media. It is complemented by a closure relation that relates the effective microscopic length to the effective packing fraction, which controls the transition from disorder to order.
\end{abstract}

Keywords: A. Fibres; B. Transport properties; C. Statistics; C. Finite element analysis (FEA); Random structure

*- Corresponding author: K. Yazdchi (k.yazdchi@utwente.nl), Tel: +31534893345, Fax: +31534893695 


\section{Introduction}

Understanding and predicting transport properties of porous media is essential in chemical, mechanical and petroleum industries [1]. This has motivated the development of relationships between macroscopic parameters, like permeability, and microstructural parameters, like fibre arrangements, shape and orientation or tortuosity (flow path) [2].

Most porous media are particulate, but some are composed of long particles/cylinders and, therefore, may be considered as fibrous media. Common examples of fibrous media include composite materials, industrial filters, biological tissues, etc.

Resin transfer molding (RTM) is an efficient and frequently used process for producing fibre reinforced polymer composites with simple or complex shapes. The permeability is essential in such process and can be determined by various methods, e.g., experimental measurements, numerical calculations or analytical predictions. Experimental measurements often require a large number of carefully controlled experiments and are normally expensive. Analytical predictions based on theoretical assumptions (and validated by experiments and/or numerical studies) are often applicable in a certain range of fibre volume fraction only.

With the recent progress in computational and numerical tools, one can now predict the macroscopic properties of fibrous materials with rather complex microstructure. Chen and Papathanasiou [3-4] computationally investigated the flow across randomly distributed unidirectional arrays using the boundary element method (BEM) and found a direct correlation between permeability and the mean nearest inter-fibre spacing. Papathanasiou [5] performed a similar study for unidirectional square arrays of fibre clusters (tows) using the BEM. He showed that the effective permeability of assemblies of fibre clusters depends strongly on the intra-tow porosity only at low values of the inter-tow porosity (high density). Song et al. [6] calculated the permeability tensor for a 3D circular braided preform by solving a boundary problem of a periodic unit cell. The flow field through the unit cell is then obtained by using a 3D finite volume method and Darcy's law is utilized to obtain the permeability tensor. Their numerical results show that the permeability in the machine direction of the preform was the highest among three directions. Takano et al. [7] employed an asymptotic homogenization theory to evaluate 
the permeability of woven fabrics with the help of finite element method (FEM). They investigated the effect of woven architecture on the permeability characteristics for plainwoven fabrics with and without shearing deformation. In our recent study [8], the effect of several microstructural parameters such as fibre shape and orientation on the macroscopic permeability of $2 \mathrm{D}$ regular fibrous media was investigated using a large set of FEM simulations.

The permeability of ordered (regular) fibrous media is known to be a deterministic function of their porosity in the limit of large and small volume fractions. However, the parameters affecting the permeability of disordered (random) fibre arrays are not very well understood. Random fibre arrays are, in principle, well suited for analysis using effective medium approaches. Based on averaging the conservation equations, Koch and Brady [9] derived a relation for the effective diffusivity coefficients in the limit of high porosity $\varepsilon$, however, that remains questionable in the porosity range of interest in composites manufacturing, e.g., $0.4<\varepsilon<0$.9. Similarly, valid for large $\varepsilon$, Drummond and Tahir [10] modeled the flow around a fibre using a unit cell approach by assuming that all fibres in a fibrous medium experience the same flow field (i.e. no interaction between them) and, therefore, the permeability can be obtained by adding the resistance of individual particles/fibres. The dependence of permeability in this limit involves logarithmic, linear and quadratic functions of the solid concentration.

Based on the lubrication approximation and assuming that the narrow gaps between adjacent cylinders dominate the flow resistance for small $\mathcal{E}$, Gebart [11] presented an expression for the transverse permeability of square or hexagonally ordered arrays. $\mathrm{He}$ found that the dependence of permeability on fibre volume fraction in this limit is a power law.

The earliest and most widely applied models in the composites literature, i.e., intermediate porosity regimes, for predicting permeability are capillary models such as the Carman-Kozeny (CK) equation [12] where a constant (Kozeny constant) is supposed to account for the structure at different porosities. While some studies have reported success with this relation [13], discrepancies are also reported [14]. Results from numerical modeling [3] and experimental studies [15] indicate that, at best, capillary models represent the behavior of fibrous materials over a limited porosity range. The 
value of the 'Kozeny constant', however, is usually quite different from its theoretical value for idealized systems like random sphere packings. In essence, a function of porosity alone is not sufficient to explain the observed variability in permeability data. The fact that the Kozeny constant needs to be experimentally measured, limits further the usefulness of this model for predictive purposes. For sedimentary rocks, especially sandstones, Katz and Thompson [16] found, using percolation theory, a power law relation between the macroscopic permeability and microstructural descriptors for rocks, i.e. the critical pore diameter. To our knowledge, relation between microstructure and macroscopic permeability of the fibrous medium, like composite materials, has not been studied systematically.

The objective of this study is to computationally investigate the effects of microstructural parameters such as fibre arrangements on the macroscopic permeability by using a FEM for fluid flow over a wide range of porosity. To this end, the macro description of fluid flow equations and the numerical tool employed to solve these equations are presented in Section 2. Volumes of different sizes, formed by randomly placed non-overlapping arrays of parallel cylinders perpendicular to the flow direction, are constructed in four different ways in Section 3. The size effect, the homogeneity and the isotropy of the fibre arrangements are analyzed using several statistical tools. In Section 4, we present a microstructural model based on the lubrication effect of the narrow channels as an attempt to (i) combine our various simulations in a wide range of porosity and (ii) relate the micro to the macro properties of fibrous materials. The paper is concluded in Section 5 with a summary and outlook for future work.

\section{Mathematical formulation and methodology}

This Section is dedicated to the macroscopic description of the flow equations applied in fibrous structures and our methodology, e.g., the method of discretization of the domain and boundary conditions applied to our FEM based simulations.

\subsection{Macroscopic description of the flow equations}


The velocity and pressure profiles through the fibrous media can be obtained from the solution of the conservation laws, namely, the continuity equation (conservation of mass) and the Navier-Stokes (NS) equations (conservation of momentum). In the absence of body forces and for Newtonian fluid, incompressible, steady state flow, the equations of conservation of mass and momentum are:

$$
\begin{aligned}
& \nabla \cdot u=0, \\
& \rho(u \cdot \nabla u)=-\nabla p+\mu \nabla^{2} u .
\end{aligned}
$$

where $\rho, u, p$ and $\mu$ are density, velocity, pressure and viscosity of the fluid, respectively. According to Darcy's law for unidirectional flow through a porous medium in the creeping flow regime, the superficial fluid velocity $U=\frac{1}{V} \int_{V_{f}} u d v\left(V, V_{f}\right.$ are the total volume and volume of the fluid) is proportional to the pressure gradient:

$$
U=-\frac{K}{\mu} \nabla p .
$$

The proportionality constant $K$, is called the permeability of the medium, which strongly depends on the microstructure (such as fibre arrangement, void connectivity and inhomogeneity of the medium) and also on porosity. By increasing the pressure gradient, one can observe a typical departure from Darcy's law (creeping flow) at sufficiently high Reynolds number, Re $>0.1$ [17]. In order to correctly capture the influence of the inertial term, Yazdchi et al. [17] showed that the original Darcy's Law can be extended with a power law correction with powers between 2 and 3 for regular structures. Koch and Ladd [18] simulated moderate Reynolds number flows through periodic and random arrays of aligned cylinders. They showed that the quadratic inertial effect became smaller at higher volume fractions. Similarly, Hill et al. [19-20] examined the effect of fluid inertia in cubic, face-centered cubic and random arrays of spheres by means of lattice-Boltzmann simulations. They found good agreement between the simulations and the Ergun correlation at solid volume fractions approaching the closely-packed limit at moderate Reynolds number $(\operatorname{Re}<100)$. However, in the following, we restrict ourselves to creeping flow regime, i.e. $\operatorname{Re} \ll 1$.

\subsection{Computational method}


The FEM software ANSYS ${ }^{\circledR}$ is used to calculate the fluid velocity from discretization of Eq. (1) into linear triangular elements. They were then solved using the segregated, sequential solution algorithm. This means that element matrices are formed, assembled and the resulting systems solved for each degree of freedom separately. Some more technical details are given in Ref. [8]. Afterwards, the superficial velocity and, using Eq. (2), the permeability of the fibrous material is obtained. Fig. 1 shows a 3D/2D representation of 200 randomly distributed fibres normal to the flow direction at porosity $\varepsilon=0.6$ with minimum inter fibre distance $\delta_{\min }=0.05 d$ ( $d$ is the diameter of the fibres) or the minimum dimensionless distance $\Delta_{\min }=\delta_{\min } / d=0.05$. Similar to Chen and Papathanasiou [3, 4], a minimal distance is needed in 2D to avoid complete blockage. The microstructural parameters, namely the system size, method of generation, homogeneity and isotropy of the structure will be discussed in more details in the next Section. At the left and right boundary pressure is set and at the top and bottom wall surfaces (z direction) and at the surface of the particles/fibres no-slip boundary conditions are applied. Some simulations are formed with periodic boundaries instead of walls with normal in $\mathrm{z}$ direction. Fibres are assumed to be very long so that a $2 \mathrm{D}$ solution can be applied. A typical unstructured, fine and triangular FEM mesh is also shown in Fig. 1. The mesh size effect is examined by comparing the simulation results for different resolutions. The range of number of elements is varying from $5 \times 10^{5}$ to $10^{6}$ depending on the porosity regime. The lower the porosity the more elements are needed in order to resolve the flow within the neighboring fibres, see Appendix A for more details. To obtain good statistical accuracy, the permeability values were averaged over 10 realizations.

\section{Generation of the fibrous microstructure}

Developing a model for predicting the permeability as a function of porosity and structure of the fibrous materials would help to reduce the experiments in liquid composite molding (LCM) processes such as RTM or resin infusion. Furthermore, by understanding the physics of the flow through such materials, one may tailor the microstructure such that it has both the desired reinforcing capability and also the 
permeability to be filled efficiently. To get reliable evidence and to quantify the nonuniform spatial distribution of fibres, several microstructural characteristics of fibrous material will be discussed here in detail.

Note that to ensure a gap between particles $\left(\Delta_{\min }\right)$, we assign a virtual diameter $d^{*}=d\left(1+\Delta_{\min }\right)$ to each fiber, leading to the virtual porosity $\varepsilon^{*}=1-(1-\varepsilon)\left(1+\Delta_{\min }\right)^{2}$, see Fig. 1, which has been used for packing generation in the rest of this section.

\subsection{Generating an appropriate random structure}

A representative volume element (RVE) is a subdivision of the material that has properties consistent with those of the whole system. It plays a central role in the mechanics and physics of random media with a goal to predict their effective properties. Most of them rely on the assumption of a periodic distribution of fibres, i.e. the structures at the boundaries are similar to those in the bulk. However, realistic media are finite and confined with walls. Some systems can be very large so that boundary effects can be neglected; on the other hand, in micro-systems, the effect of the walls might show up. Therefore, unless specified otherwise, in the rest of the paper we consider a 2D representation of the fibrous composite in which the fibres are randomly distributed in a square domain and confined by walls with normal in z direction. In order to understand the wall-effects, we will vary the system size (see Appendix B for details).

\subsection{Method of generation}

To generate random, non-overlapping fibre arrays, we use different algorithms, namely, random placement (RP), a Monte Carlo (MC) procedure, an energy minimization (EM) approach and molecular dynamic (MD) simulations. Note that in all methods, we have a minimal distance $\left(\Delta_{\min }\right)$ between fibres to avoid complete blockage.

\subsubsection{Random placement (RP)}

In RP the position of fibres is randomly drawn from a uniform distribution; then this location is taken as valid if it does not overlap a previously positioned fibre. The insertion of fibres will continue until it reaches the desired number of fibres. This process leads to an asymptotic jamming limit since the space available to place successive particles 
decreases with the addition of each new particle. The minimum porosity for RP in $2 \mathrm{D}$ is estimated to be $\sim 0.447$ [21], and has found to be $\sim 0.453$ [22] via computer simulations.

\subsubsection{Monte Carlo (MC) procedure}

Given an initial fibre configuration on a triangular lattice, the MC procedure perturbs fibre centre locations in randomly chosen directions and magnitudes [3-4, 23]. The perturbation is rejected if it leads to overlap with a neighboring disk. One step consists of trying to move each disk once. We use up to $10^{6}$ steps for each realization at low porosities to get a good random configuration i.e. the MC process should generate a random position field which is short range correlated. However, at the lowest porosity, the particles remain ordered and show some dependence on the number of steps/perturbations, even for the longest simulations. For high porosities, the structures show no dependence on number of MC perturbations. Moreover, the MC algorithm can generate denser systems as compared to RP.

\subsubsection{Energy Minimization (EM) approach}

In the EM approach, infinitesimal disks are placed at random positions in the system. Then, they are gradually expanded and moved at each step to prevent particles from overlapping. When the desired porosity is reached the algorithm terminates [24]. We assume that the particles interact via the soft potential given by:

$$
V\left(r_{i j}\right)= \begin{cases}\frac{\beta}{2}\left(1-\frac{r_{i j}}{d}\right)^{2} & \text { for } \quad r_{i j}<d, \\ 0 & \text { for } \quad r_{i j} \geq d,\end{cases}
$$

where $\beta$ is the characteristic energy scale and $r_{i j}$ is the separation of particles $i$ and $j$. Potential of this form was motivated by simulation of granular materials, see next subsection, where the particles do not interact except for a strong repulsive force that keeps the particles from overlapping/deforming too much ${ }^{1}$. With this procedure, one can generate very dense systems down to $\varepsilon \sim 0.158$.

\footnotetext{
${ }^{1}$ After each expansion step, we check if any disks overlap by checking the condition $1-r_{\mathrm{ij}} / d>10^{-5}$ for each particle pair. Below this limit, the overlap is neglected. If any particles do overlap, i.e. the total energy is $E>0$, the nonlinear conjugate gradient method is used to decrease the total energy by adjusting the position
} 


\subsubsection{Molecular dynamics (MD)}

Finally, a 2D discrete element method (DEM) or soft-sphere model is used to generate a random non-overlapping disks configuration. The motion of particles is described by Newton's laws of motion. A characteristic feature of the soft-sphere models is that they are capable of handling multiple particle contacts, which are of importance when modeling dense, quasi-static systems. Particle overlaps are indicative of a collision. For all identified collisions, a contact model (here we use a simple spring/dash pot model, similar potential as Eq. (3)) is applied and the simulation is then advanced again in time [25]. The typical contact duration is:

$t_{c}=\pi / \omega, \quad$ with $\omega=\sqrt{2 k / m-\left(\eta_{0} / m\right)^{2}}$,

where $k, \omega, \eta_{0}$ and $m$ are the spring stiffness, eigen-frequency of the contact, viscous damping and mass of the particles/fibres, respectively. Note that the integration of the equations of motion is stable only if the integration time-step, $\Delta t_{\mathrm{MD}}$, is much smaller than $t_{c}$. The difference to EM is that inertia (dynamical motion of particles) is taken into account in $\mathrm{MD}^{2}$.

Having an initial fibre configuration on a regular lattice, we assign random velocities in random direction to each particle and run a MD simulation for sufficiently long time such that all particles are separated by the minimum gap $\left(\Delta_{\min }\right)$. A random structure is obtained by taking a snapshot of the final fibre positions.

In the following, we compare the statistics of the fibre arrangements generated by the four methods and investigate the influence of the packing generator algorithm on the macroscopic permeability of the medium, where [3-4] used the MC procedure only.

\subsection{Statistical analysis of the microstructure}

Various statistical descriptors have been proposed to characterize and classify microstructures based on the spatial arrangement of heterogeneities, see for example [26,

of disks so they no longer overlap $(E=0)$. Therefore, in this method, the value of $\beta$ will not affect the minimization procedure.

${ }^{2}$ Contact force parameters used in MD simulations: $k=10^{6}\left[\mathrm{~kg} / \mathrm{s}^{2}\right], m=100 \mathrm{~kg}, \eta_{0}=10^{-4}[\mathrm{~kg} / \mathrm{s}], \Delta t_{\mathrm{MD}}=10^{-4}[\mathrm{~s}]$ with the total time of simulation $t_{T}=500[\mathrm{~s}]$. 
27]. Popular among these is the radial (pair) distribution function $g(r)$, which is defined as the probability of finding the centre of a fibre inside an annulus of internal radius $r$ and thickness $\mathrm{d} r$ with centre at a randomly selected fibre. It is mathematically defined as:

$g(r)=\frac{1}{2 \pi r} \frac{\mathrm{d} K(r)}{\mathrm{d} r}$, where $K(r)=\frac{A}{N^{2}} \sum_{k=1}^{N} I_{k}(r)$.

where $K(r)$ is a second-order intensity function, also known as Ripley's function [3-4, 26] and $I_{k}(r)$ is defined as the number of centers of fibres that lie within a circle of radius $r$ about an arbitrarily chosen fibre and $N$ is the number of fibres in the observation area $A$. Given a Poisson point distribution, the complete randomness of the fibre distribution will assure that $g(r)=1$ (with some fluctuations) for all distances considered. A statistically valid fibre distribution without long-range order will have $g(r)$ tending to 1 when the distance $r$ increases. The comparison of $g(r)$ for packings generated with different methods is shown in Fig. 2(a). In this graph $r$ varies from $d$ (diameter of the fibre) to approximately $1 / 3$ of the sample size to avoid the boundary (edge) effect on the statistics. Fig. 3 shows the actual area and the center area which we used to calculate $g(r)$, in red color, for various creation methods. In all methods we have 800 fibres at constant porosity $\varepsilon=0.6$ with minimum inter fibre distance $\Delta_{\min }=0.05$. Local maxima indicate the most frequent distances and local minima correspond to the least frequent distances between pairs. The first (highest) peak in the graph is caused by the physical area (excluded "volume") of the fibres with virtual diameter $d^{*}$. For larger $r$, we observe a number of oscillations until $g(r)$ approaches the value of unity indicating the numerically generated microstructures are statistically random for larger $r$. The EM method has the largest peak at $r / d \sim 1.05$ (minimum allowable inter fibre distance) and the most rapid decay with distance, followed by a second peak at $r / d \sim 2.1$ (equivalent to $r / d^{*} \sim 2$ ), which is an indication of fibre agglomeration (or a clustered structure). For the RP algorithm the oscillations around the complete randomness value of $g(r)=1$ are most damped compared to the other methods, however, the location of local maxima/minima is almost the same as for MD and MC, which lead to the most similar $g(r)$ among the four methods. For configurations with more fibres and different porosities (data not shown), qualitatively, the same trends are observed. 
The fibre arrangement has a direct influence on the effective properties of the medium. In Fig. 2(b) the variation of the normalized permeability $K / d^{2}$, as a function of the number of fibres $N$, is shown. As expected, the packings generated with $\mathrm{MC}$, MD or RP (with similar fibre distribution) have practically the same permeability, especially for larger $N$. However, the clustered structure (generated by EM) has a lower permeability for all numbers of fibres. This is due to the fact that many particles are arranged along lines sometimes with local square or triangle structure - but no evident long range order as one would have in a crystal (see Fig. 3 and the peak locations in Fig. 2(a)). This leads into more resistance to the flow (i.e. lower permeability) even at intermediate porosities (see next Section). By increasing the system size the standard deviation decreases but it remains largest - for EM - indicating correlations built into the method. For most data presented in Section 4, we stick to the MC procedure that generates the structures similar to what is observed in real composite manufacturing processes [23] and since it is faster than the MD method. More details on the effect of system size and boundary conditions on statistical descriptors and macroscopic permeability are provided in Appendix B.

\subsection{Isotropy and homogeneity of the packing}

Since the media studied here consist of randomly distributed fibres, they are expected to be isotropic (no preferential flow direction). Therefore, the normalized permeability as a function of porosity in both horizontal and vertical directions, as shown in Fig. 4, is independent of flow direction. As mentioned before, the EM approach tends to generate clustered packings unlike the MC procedure (or MD simulations, which create more homogenous structures). Fig. 4 shows that all methods (namely MC, MD and EM) create isotropic media (with respect to horizontal and vertical flow) for all porosities. For comparison, the numerical results of Sangani and Yao [28] and Chen and Papathanasiou [3] for transverse flow are also included in Fig. 4. The homogeneity of the packing has negligible effect on permeability at high porosity $(\varepsilon>0.65)$, however, at lower porosities the clustered structure has lower permeability as compared to the homogenous configuration. The reason is that for dilute fibrous media there is no correlation between the solid fibre bundles, however, at lower porosities in the packing generated with the EM approach, we see local fibre clusters, which tend to block the channel and cause a 
drop in permeability. This was confirmed by studying the velocity fields (not shown here) and is also visible in the PDF of neighbor distances.

\subsection{Effect of minimum inter-fibre distance $\left(\Delta_{\min }\right)$}

The minimum inter-fibre distance $\left(\Delta_{\min }\right)$ was taken as $5 \%$ of the "true" fibre diameter, $d$ in our simulations up to now to avoid complete blockage in 2D. In the following we scale the permeability values such that they collapse on a single curve valid for all values of $\Delta_{\min }$ ranging from 0.2 to 0.005 . Note that the lower the $\Delta_{\min }$ the more elements are needed to resolve the flow in the gaps between fibres.

Fig. 5 shows the effect of $\Delta_{\min }$ on the fibre arrangements and fluid velocity. Note that large values of $\Delta_{\min }$ lead to local (triangular) ordering (Fig. 5(a)), whereas small $\Delta_{\text {min }}$ result in patterns showing local fibre aggregation (Fig. 5(b)). We observe stagnancy of the fluid between fibre aggregates or within rings of close-by fibres, while a few major flow paths with relatively high flow speed exist in all configurations with disorder.

The permeability, $K / d^{2}$, for small porosity (i.e. the maximum random close packing fraction $\sim 0.84$ ( $\varepsilon_{c}^{r}=0.16$ ) [29], see the Appendix C.) saturates at an a-priori finite value. Larger minimal distances $\Delta_{\text {min }}$ lead to over-proportionally larger permeability (plot not shown). When the data are scaled by the permeability expected for a periodic hexagonal

cell as $\left(K-K_{\Delta_{\min }}^{\text {hex }}\right) / d^{2}$, see Ref. [8], the data vanishes at a finite porosity, which decays with decaying $\Delta_{\min }$. Therefore, we conclude that the minimal permeability of our random structure is somewhat lower than the one of a hexagonal lattice (see the inset in Fig. 6). In other words, the systems with more uniform and weak channels (Fig. 5(a)) have higher permeability than the systems with fewer dominant channels (Fig. 5(b)).

Fig. 6 shows the effect of $\Delta_{\min }$ on the normalized permeability - after scaling with an appropriate permeability $K^{\mathrm{c}} / d^{2}$ that is obtained by multiplying the $K_{\Delta_{\min }}^{\text {hex }} / d^{2}$ with a prefactor such that all the data collapse onto a single curve, i.e. Eq. (D.3) in appendix D. The minimal permeability for disordered fibre-arrays can then be cast into a formula: $K^{c} / d^{2} \simeq \eta K_{\Delta_{\min }}^{\text {hex }} / d^{2}$, with factor $\eta=\left(1+\Delta_{0} / \Delta_{\text {min }}\right)$ and $\Delta_{0}=0.14$ (fitting parameter) that accounts for the minimal inter fibre distance. The numerical values of the 
critical porosity, $\varepsilon_{\Delta_{\min }}^{\text {hex }}$ and permeability $K_{\Delta_{\min }}^{\text {hex }} / d^{2}$ (for a perfectly hexagonal lattice) and corrected permeability, $K^{\mathrm{c}} / d^{2}$ at different $\Delta_{\min }$ are given in Table 1 . The scaling factor, $\eta$ was obtained by fitting the $K^{\mathrm{c}} / K_{\Delta_{\min }}^{\text {hex }}$ ratios at different $\Delta_{\min }$, using a least square approach. With increasing $\Delta_{\min }$ the scaling factor decreases towards unity and the corrected permeability values, $K^{\mathrm{c}} / d^{2}$, approach the hexagonal cell values. Note that the last data point at each $\Delta_{\min }$ branch is slightly below $\varepsilon_{\text {order }}^{*} \cong 0.3$ (see the arrows). Because all $\varepsilon^{*}>\varepsilon_{\text {order }}^{*}$ can be considered as random (almost), whereas $\varepsilon^{*}<\varepsilon_{\text {order }}^{*}$ are partially ordered, we exclude the latter. The inset in Fig. 6 shows the zoom of permeability data before scaling at low porosities together with the perfectly hexagonal values obtained from lubrication theory [11] (red dashed line) and finite element results [8] (blue stars). The permeability of random fibre arrangements tends to be smaller than for hexagonally ordered arrays. In the next section, our attempt is to extend the lubrication theory for ordered arrays [11] into random configurations.

\subsection{Summary}

In summary, the random generator algorithm can affect the local fibre distribution especially at low porosities. All methods used in this study generate isotropic structures with respect to vertical and horizontal direction. The EM approach used has created more heterogeneous packings compared to other methods.

Note that in general, the optimum number of particles (as small as possible but large enough to represent bigger samples) depends on the porosity. Periodic boundary conditions can reduce this number since inhomogeneity at the walls is removed (see Appendix B). As standard $N=800$ was applied and, as before, the permeability is calculated on the center part of the system (see Fig. 3).

Putting an artificial gap between fibres $\left(\Delta_{\min }\right)$ changes the microstructure and accordingly the permeability of the packing, at high volume fractions (small $\varepsilon$ ). As the main result of this section, correcting the permeability values with the empirical minimal permeability for random structures, $K^{\mathrm{c}} / d^{2}$, leads to perfect scaling (standard deviation discrepancy less than 5\%) of all random/disordered structures data for all permeabilities, valid for all 
$\Delta_{\min }$, as shown in Fig. 6. Understanding the microscopic origin of this scaling is the subject of the next section.

\section{Theoretical prediction of the permeability}

In this section we present a microstructural model for predicting the macroscopic permeability based on the lubrication effect of the narrow/effective channels.

\subsection{Statistical characterization of effective channels}

Several statistical and structural descriptors, i.e., Delaunay triangulation (DT), Delaunay edges (DE), hydraulic diameter $\left(D_{h}\right)$ and fibre/particle nearest neighbor distances $\left\langle D_{n}\right\rangle$, obtained from fibre distributions, are discussed here and used to characterize the narrow channels.

\subsubsection{Nearest neighbor distances}

Here we define the mean value of the $n$-th nearest neighbor distances $\left\langle D_{n}\right\rangle$ normalized with the diameter of the fibres $\gamma_{n}=\left(\left\langle D_{n}\right\rangle-d\right) / d$. The diameter, $d$ can be expressed in terms of macroscopic porosity as $d=\sqrt{4(1-\varepsilon) /(\lambda \pi)}$ where $\lambda=(1-\varepsilon) / V_{p}$ is the number density (number of fibers per unit area) equal to 2 (800 fibres in a box of $20 \times 20$ $\left.\left[\mathrm{m}^{2}\right]\right)$ in our simulations. Similarly, one can define the effective normalized $n$-th nearest neighbor distances as $\gamma_{n}^{*}=\left(\left\langle D_{n}\right\rangle-d^{*}\right) / d^{*}$ with $d^{*}=d\left(1+\Delta_{\min }\right)$. The former, $\gamma_{n}$, quantifies the channel width available for flow, while the latter is a measure for the effective distance due to the minimum inter fibre distance, which is relevant for microstructure but not so much for fluid flow.

Fig. 7(a) and (b) show the $2^{\text {nd }}$ and $1^{\text {st }}$ nearest neighbor distances, respectively. Note that the network in Fig. 7(b) appears considerably more dilute than that in 7(a). While not percolating separately $(a, b)$, the first and second neighbor network does percolate. Flow does less likely go through the narrowest gaps, but there are enough $2^{\text {nd }}$ channels, so that the second neighbor distances should control the flow. 


\subsubsection{Normalized hydraulic diameter $\left(D_{h} / d\right)$}

Another measurable quantity that is frequently used in modeling of porous/fibrous structures is the hydraulic diameter $D_{h}$ [1]. When one has obstacles like fibres (or particles) instead of straight pores, the hydraulic diameter can be defined as:

$D_{h}=\frac{4 \varepsilon V}{S_{v}}=\frac{4 \varepsilon}{(1-\varepsilon) a_{v}}=\frac{\varepsilon d}{(1-\varepsilon)}, \quad$ with $a_{v}=\frac{\text { particle surface }}{\text { particle volume }}=\frac{S_{v}}{(1-\varepsilon) V}=\frac{4}{d}$,

with the total volume of the unit cell, $V$, the total wetted surface, $S_{v}$, and the specific surface area, $a_{v}$. Note that the hydraulic diameter, in this way, is expressed as a function of the measurable quantities porosity and specific surface area. The above value of $a_{v}$ is for circles (cylinders) - for spheres one has $a_{v}=6 / d$. Therefore the relation between normalized hydraulic diameter $D_{h} / d$ and porosity for fibres will reduce to:

$\frac{D_{h}}{d}=\frac{\varepsilon}{(1-\varepsilon)}$

Note that in the following the hydraulic diameter, even though it could be defined per particle or per Delaunay triangle, will only be used as averaged quantity.

\subsubsection{Delaunay triangulation (DT)}

A Delaunay triangulation (DT) is the set of lines joining a set of points such that each point is joined to its nearest neighbors [30]. It satisfies an "empty circle" property, i.e. the circumcircle of each triangle (formed by three points) does not contain any of the other points. It is the dual graph of the Voronoi diagram (VD) and has a node (fibre center) for every Voronoi cell and an edge between two nodes if the corresponding cells share an edge (see Fig. 7(c), the blue lines show the DT edges). This concept is suitable for the characterization of the arrangement of dispersed fibres [31]. The DT has many other applications such as finding the nearest neighbor, mesh generation and surface reconstruction, interpolation and extrapolation, strain calculation [32], etc. Here we used the statistics of the Delaunay edges as a descriptor to characterize the spatial dispersion of fibres. One average quantity is the mean value of all DT edge lengths $\left\langle e_{D T}^{p}\right\rangle$ normalized with the diameter of the fibres $d$, i.e. $\gamma_{D T}^{p}=\left(\left\langle e_{D T}^{p}\right\rangle-d\right) / d$. For a perfect triangular lattice 
it reduces to exactly the inter fibre (surface-to-surface) distance and for the lowest porosity $\varepsilon_{\text {hex }}^{*}=(1-\pi /(2 \sqrt{3}))$ one has $\gamma_{D T}^{p}=\Delta_{\min }$.

Similarly, one can find the shortest Delaunay edges for each particle and then average over all particles, i.e., the first, $\gamma_{1}^{p}=\left(\left\langle e_{1}^{p}\right\rangle-d\right) / d$, the second, $\gamma_{2}^{p}=\left(\left\langle e_{2}^{p}\right\rangle-d\right) / d$, etc. Our numerical results show that $\gamma_{1,2,3}^{p} \cong \gamma_{1,2,3}$ (for $\gamma_{1}^{p} \cong \gamma_{1}$ see Fig. 8).

\subsubsection{Delaunay edges (DE)}

For a given Delaunay triangulation the local mass conservation implies that in steady state condition the net flow through all the DT edges belonging to one triangle is zero. Therefore, the characteristic length of these edges might also be useful to describe the macroscopic flow field.

We define $\gamma_{1}^{T}$ as the mean value of the shortest Delaunay edges $\left\langle e_{1}^{T}\right\rangle$, (averaged over Delaunay triangles and not fibres) normalized by the fibre diameter, $\gamma_{1}^{T}=\left(\left\langle e_{1}^{T}\right\rangle-d\right) / d$. Fig. 7(d) shows these shortest edges. The red color shows the repeated edges of neighboring triangles. Note that the superposition of the network in Fig. 7(a) and (b) is very similar to (d) - not shown here. The shortest Delaunay triangle edges form a percolated edge-network, where empty "channels" indicate the regions (channels) in which the fluid is most likely flowing (fast).

Fig. 8 shows the variation of all these descriptors as a function of porosity. The normalized mean nearest neighbor distances approach the minimum inter fibre distance (i.e. $\left.\Delta_{\min } \sim 0.05\right)$ at low porosities (locally crystalline structure). On the other hand, at high porosities we are reaching the analytical values of random point patterns at $\varepsilon=1$.

As expected, the values obtained by averaging the shortest Delaunay edges for each fibre $\gamma_{1}^{p}$, match the results of nearest neighbor distances $\gamma_{1}$. Interestingly, we observe that the mean values of the $2^{\text {nd }}$ nearest neighbors $\gamma_{2}$ (red squares) approximately match the values of the shortest DT edges $\gamma_{1}^{T}$ (averaged over triangles). So far we have not found a mathematical proof for this observation. For regular (i.e. square or hexagonal) arrays, the statistical descriptors are the same $\left(\gamma_{1}=\gamma_{2}=\gamma_{3}=\gamma_{1}^{T}\right)$ and $\gamma_{1}^{T}$ is thus a deterministic 
function of porosity, see next section. Interestingly the values of $\gamma_{D T}^{p}$ for random and hexagonal arrays are almost the same, showing that the mean value of neighboring fibre distances obtained by averaging over all DE does not depend much on the structure. Another interesting observation is that for $\varepsilon<0.8$ the normalized hydraulic diameter $D_{h} / d$ has also the same trend as the shortest Delaunay edges and the $2^{\text {nd }}$ nearest neighbor distances $^{3}$ and, when scaled by a factor $1 / 6$, even agrees quantitatively well.

\subsubsection{Microscopic channel width in terms of macroscopic porosity}

Based on a least square fit approach, an empirical expression is obtained for the mean values of the $n$-th nearest neighbor distances, $\gamma_{n}$ as functions of porosity:

$\gamma_{n}=\left\langle D_{n}\right\rangle / d-1 \quad$ with $\quad \frac{\left\langle D_{n}\right\rangle}{\left\langle D_{n}^{p}\right\rangle}=1+\varphi_{n}\left(\frac{1-\varepsilon}{1-\varepsilon_{c}^{r}}\right)^{\xi_{n}}$

where $\varepsilon_{c}^{r}\left(\Delta_{\min }\right)$ and $\left\langle D_{n}^{p}\right\rangle$ are the corresponding critical porosity of a random packing with $\varepsilon_{c}^{*} \cong 0.16$ and mean nearest neighbor distance for random points calculated analytically from Eq. (B.1), respectively. The quantities $\varphi_{n}$ and $\xi_{n}$ are fitting constants for a given $n$ and, in general, weakly depend on $\Delta_{\min }$. The numerical values of $\left\langle D_{n}^{p}\right\rangle, \varepsilon_{c}^{r}$, $\varphi_{n}$ and $\xi_{n}$ for $n=1,2,3$ and various $\Delta_{\min }$ are given in Table 2 . Thus, one can easily estimate the $n$-th nearest neighbor distances of hard disc packings by only knowing its macroscopic porosity. Fig. 9 shows the variation of mean values of the $2^{\text {nd }}$ nearest neighbors, $\gamma_{2}$ as function of porosity together with the best fits, Eq. (8), at different $\Delta_{\min }$. At high porosities, $\Delta_{\min }$ has less effect on $\gamma_{2}$, however, by decreasing porosity, $\gamma_{2}$ has larger values at higher $\Delta_{\min }$ and approaches the limit value $\gamma_{2}=\Delta_{\min }$. The inset of Fig. 9 shows that by plotting the effective $\gamma_{2}^{*}=\left\langle D_{2}\right\rangle / d^{*}-1$ against the effective porosity $\varepsilon^{*}$, all data collapse and one gets the universal curve in Eq. (8) with $\varepsilon_{c}^{r} \cong 0.16, \varphi_{2} \cong 0.35$ and $\xi_{2} \cong 0.7$ corresponding to $\Delta_{\min }=0$, i.e. $\gamma_{2}^{*}=\gamma_{2}$ and $\varepsilon^{*}=\varepsilon$.

\footnotetext{
${ }^{3}$ This may explain the limitation/failure of capillary models such as the Carman-Kozeny (CK) equation at high porosities which are based on the hydraulic diameter concept.
} 
One of our hypotheses is that the percolated network of the shortest (triangle) edges, $\gamma_{1}^{T} \cong \gamma_{2}$, controls the overall drag (permeability) of the fibrous material (which is confirmed a-posteriori by our numerical results below). However, the microstructure is controlled by $\gamma_{2}^{*}$ which leads to larger excluded volume during packing generation. In the next subsection we show that, similar to regular fibre arrays [11], these channels between triangles are correlated with the macroscopic permeability of the porous medium for a very wide range of porosities.

\subsection{Permeability prediction in terms of effective channels}

Based on the Navier-Stokes equation, Gebart [11] derived the permeability of an idealized unidirectional reinforcement consisting of regularly ordered, parallel fibres both for flow along and for flow perpendicular to the fibres. The solution for flow along fibres has the same form as the CK equation [12], while the solution for transverse flow has a different form as:

$$
\frac{K}{d^{2}}=C\left(\sqrt{\frac{1-\varepsilon_{c}}{1-\varepsilon}}-1\right)^{2.5},
$$

where $\varepsilon_{c}$ is the critical porosity below which there is no permeating flow and $C$ is a geometric factor $\left(C=\frac{4}{9 \pi \sqrt{2}} \cong 0.1, \varepsilon_{c}=1-\frac{\pi}{4} \cong 0.2146\right.$ for a square array and $C=\frac{4}{9 \pi \sqrt{6}} \cong 0.0578, \varepsilon_{c}=1-\frac{\pi}{2 \sqrt{3}} \cong 0.0931$ for a hexagonal array [8]). Gebart [11] presents numerical results, obtained using a finite difference solution of the NS equations that show excellent agreement with Eq. (9) up to porosities of $\sim 0.65$.

In order to rewrite Eq. (9) in terms of $\gamma_{2}$, we express the porosity as function of the lattice distance $a$ and fiber-diameter $d$ :

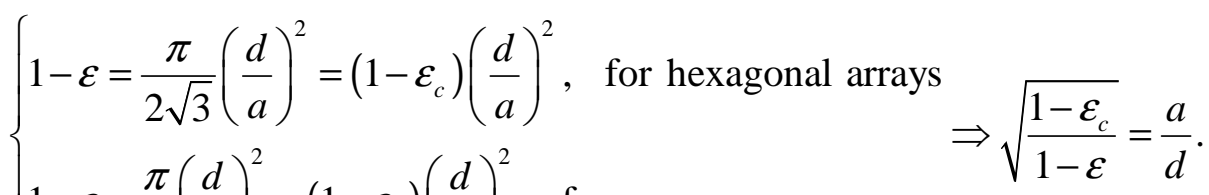


For regular arrays $\gamma_{1}=\gamma_{2}=\gamma_{3}=\gamma_{1}^{T}$, whereas for random arrays $\gamma_{2} \simeq \gamma_{1}^{T}$ (see Fig. 8), so that $\gamma_{2}=\left\langle D_{2}\right\rangle / d-1$ can be written in terms of the lattice distance as:

$$
\gamma_{2}=\left(\frac{a}{d}\right)-1
$$

Inserting Eq. (11) into Eq. (10) and combining it with Eq. (9), leads to:

$$
\frac{K}{d^{2}}=C \gamma_{2}^{2.5} \text {, }
$$

as exactly valid for regular square or hexagonal arrays at low and moderate porosities with corresponding $C$, see above.

In this representation, the normalized permeability scales with the (for example $2^{\text {nd }}$ ) narrowest channels, i.e., $\gamma_{2}$, as a power law with power 2.5. Relation (12) is remarkable, since it enables one to accurately determine the macroscopic permeability of a given packing just by measuring the $2^{\text {nd }}$ narrowest channels, i.e., $\gamma_{2}$, from particle positions or the narrowest Delaunay edges, i.e., $\gamma_{1}^{T}$, from Delaunay triangles. Below, we numerically confirm the validity of Eq. (12) for both regular and also random configurations.

The shortest Delaunay edges and the $2^{\text {nd }}$ neighbor distances practically coincide and form the network of channels through which the flow must go. Therefore we expect that the parameter, which characterizes the system and correlates with the permeability, is $\gamma_{1}^{T}$ or $\gamma_{2}$. Fig. 10 shows the variation of the normalized permeability as a function of the statistical descriptors discussed in Sections 3. The macroscopic permeability almost correlates with the shortest Delaunay triangle edges as a power law, similar to Eq. (12) for regular arrays, in a wide range of porosity. The solid blue line is the best power law fit (with fixed power 2.5$)$. The universal random configuration pre-factor $(C \sim 0.2)$ seems to be only weakly dependent on the minimum inter fibre distance, data not shown. With decreasing porosity the data deviate from the solid line showing the appearance of ordering in the structure. By correcting Eq. (12) as

$\frac{K}{d^{2}}=C \gamma_{2}^{2.5} \chi\left(\gamma_{2}\right) \quad$ with $\quad \chi\left(\gamma_{2}\right)=\left(1-\chi_{0} e^{-m \gamma_{2}}\right), \chi_{0} \cong 0.5, m \cong 3$.

we present a universal law for predicting the macroscopic permeability in terms of $\gamma_{2}$ (with Eq. (8) as closing relation with porosity) in a wide range of porosities 
$\left(0.3<\varepsilon^{*}<0.95\right)$ for disordered arrays. The exponential correction factor, $\chi\left(\gamma_{2}\right)$ was obtained by least square fitting the ratio between numerical data and Eq. (12) and accounts for (partial) ordering effects. This observation is remarkable as it indicates that the Gebart lubrication theory (Eq. (12)), originally obtained for dense ordered arrays, is also valid for random arrays in moderate and dilute regimes by using the $\gamma_{2}$ or $\gamma_{1}^{T}$ as the effective channel width. Fig. 11 shows the variation of the normalized permeability as function of $\gamma_{2}$ at different values of $\Delta_{\min }$ together with the proposed closed form relations in Eqs. (12) and (13). In contrast to Fig. 6, here, the permeability data are not corrected by $K_{c}$, but are collapsed as the microscopic effective channel width $\gamma_{2}=\gamma_{2}\left(\gamma_{2}^{*}\right)$ takes care of the effect of $\Delta_{\min }$. For all values of $\Delta_{\min }$, Eq. (13) correctly predicts the macroscopic permeability with maximum deviation of $10 \%$ for $\varepsilon^{*}>0.3$. More discussion on very dense regimes, i.e. $\varepsilon^{*}<0.3$, where we have long range correlations due to partial up to strong ordering, is given in Appendix C. For comparison, the analytical prediction for ordered arrays (square and hexagonal configurations), i.e. Eq. (12) with the same power 2.5 but different constants $C$, are also shown.

\section{Summary and conclusions}

A finite element method (FEM) based model has been employed to calculate the transverse permeability of random fibrous media composed of long unidirectional cylinders/fibres for a wide range of porosity. The microstructure of the fibrous media has been characterized using the pair distribution function and neighbor distance statistics. Providing information about short range correlations, these microstructure descriptors allow us to characterize the spatial heterogeneity of the fibre structures, construct computer generated microstructures for further simulation, or correlate the material microstructure to macroscopic properties as, e.g., permeability. The conclusions from our statistical analysis of the microstructures and the permeability are:

- For relatively large systems, the packings obtained from different random generator algorithms are isotropic and homogenous (far away from the walls). Their properties are similar and independent of the system size, except for the 
energy minimization (EM) approach, which generates clustered structures. Periodic boundary conditions reduce the minimum required number of fibres to reach size-independence.

- By increasing porosity, the PDF of nearest neighbor distances will change from exponential to Gaussian, as relevant for random point patterns only.

- The packings with higher inter fibre distance, $\Delta_{\min }$, have more uniform and weaker flow channels and therefore higher permeability and the behavior is determined by $\varepsilon^{*}=f\left(\varepsilon, \Delta_{\text {min }}\right)=1-(1-\varepsilon)\left(1+\Delta_{\text {min }}\right)^{2}$.

- All random structure permeability data (for all studied minimal inter-fibre distances) are scaled by subtracting the random packing minimal permeability $K^{c} / d^{2} \simeq \eta K_{\Delta_{\min }}^{\text {hex }} / d^{2}$ that is proportional to the equivalent minimum of a regular structure and a pre-factor that increases with decreasing minimal distance. The low porosity random regime cannot be reached, since partial ordering sets in below a certain threshold $\left(\varepsilon^{*} \cong 0.3\right)$.

Based on the lubrication effect of the narrow channels, we found a universal power law relationship between the permeability values obtained from fluid flow simulations and the microscopic mean values of shortest Delaunay triangulation edges constructed on the fibre center positions. From the microscopic point of view, our numerical results show that the mean values of the $2^{\text {nd }}$ nearest neighbors $\gamma_{2}=\left(\left\langle D_{2}\right\rangle-d\right) / d$ (averaged over all fibres) match the values of shortest DT edges $\gamma_{1}^{T}=\left(\left\langle e_{1}^{T}\right\rangle-d\right) / d$ (averaged over all triangles). Astonishingly, the proposed power law is valid for both ordered and disordered arrays at all porosities, given a correction dependent only on $\gamma_{2}$. The superposition of $1^{\text {st }}$ and $2^{\text {nd }}$ nearest neighbor channels forms a similar percolated network as the shortest DT edges, with average $\gamma_{1}^{T}$, which dominate the flow since they represent the fluid channels through which the flow must go (with preference for the wider $2^{\text {nd }}$ neighbor channels).

In summary, a closed form relation for predicting the macroscopic permeability for ordered/disordered fibre arrangements is observed in terms of the microstructural average 
channel width $\gamma_{2}$ as $K / d^{2}=C \gamma_{2}^{2.5}\left(1-\chi_{0} e^{-m \gamma_{2}}\right)$, valid for wide range of porosities and all values of inter-fibre distance $\Delta_{\min }$. Note that Eq. (8) relates $\gamma_{2}=\gamma_{2}\left(\gamma_{2}^{*}\right)$ with $\gamma_{2}^{*}=\gamma_{2}^{*}\left(\varepsilon^{*}\right)$ and $\varepsilon^{*}=\varepsilon^{*}(\varepsilon)$ to macroscopic porosity and therefore closes this relation.

The results obtained in this study and the general relationships proposed for the permeability, can be utilized for composite manufacturing, e.g. resin transfer moulding processes. Furthermore, our results can be used for validation of advanced models for particle-fluid interactions in a multi-scale coarse graining approach, as carried out in our ongoing work. By analogy, the permeability in 3D random packings should depend on the smallest faces of Delaunay tetrahedrons $\gamma_{1}^{T 3 D}$, possibly with the chance for similar unique scaling relations as in $2 \mathrm{D}$, a prediction that waits for numerical/experimental proof.

\section{Acknowledgements:}

The authors would like to thank M. van der Hoef, A. J. C. Ladd, C. S. O'Hern, X. Chen, K.W. Desmond and A.R. Thornton for helpful discussion and acknowledge the financial support of STW through the STW-MuST program, project number 10120.

\section{Appendix A. Mesh sensitivity analysis}

Due to the difference in scale between domain size and gap size between neighboring fibers, this typically requires local mesh refinement. For different porosities, flow through random fiber arrangements was simulated at different mesh resolutions (number of elements, $N_{e}$ ). The dependence of the solution in terms of the calculated normalized permeability at dense, $\varepsilon=0.4$ (in blue) and dilute, $\varepsilon=0.8$ (in red) regimes is shown in Fig. A1. At larger porosities (dilute systems) less number of elements would be sufficient to get convergent solution. Our numerical results show that in all simulations we need at least $\sim 10$ rows of elements between neighboring particles to correctly capture the fluid behavior and obtain a converging solution.

Appendix B. Study of the system size (edge) effects 
The random fibrous structure should be large enough to capture the microscopic properties and/or the flow characteristics in the matrix. Increase in the system area implies a linear increase in the number of grid points in the computational mesh. Therefore, we need to find an optimum system size. Related to this, Grufman and Ellyin [33] determined a representative volume element size for composite laminate by applying the Kolmogorov goodness-of-fit test. Du and Ostoja-Starzewski [34] studied the finitesize scaling trend to RVE of the Darcy law for Stokesian flow in random porous media without invoking any periodic structure assumptions, but only assuming the microstructure's statistics to be spatially homogeneous and ergodic. They show that the higher the density of random disks, the smaller the size of RVE pertaining to Darcy's law. Trias et al. [35] show that the minimum system size for typical carbon fibre polymer composites is $\Omega=L / d=25$ (where $d$ and $\mathrm{L}$ are the actual diameter of fibres and system length, respectively).

To study the effect of system size, we use two statistical tools, namely the pair distribution function and the nearest neighbor distance, both measure short range correlations. The so-called "structure factor" for long range correlations is not addressed here.

\section{B.1. Pair distribution function $g(r)$}

In Fig. B1 the pair distribution function $(g(r))$ is plotted for different numbers of fibres at the two cases of (a) low porosity (dense system) $\varepsilon=0.4$ and (b) dilute system $\varepsilon=0.9$. At higher porosities, we observe that by using only the center part-away from the walls/boundaries, there is no systematic size dependence concerning short range order and increasing $N$ does not create any substantial differences in $g(r)$. However, for dense systems, the correlations reach to larger and larger distances and one need a bigger system so that the order does not "reach" the walls. The exponential decrease in the local peaks of $g(r)$ at higher densities might explain the exponential distribution of nearest neighbor distances in the next subsection. The same trend was observed for periodic boundaries as the center area was used to calculate $g(r)$ (data are not shown here). 
Note that by knowing the optimum number of fibres, one can easily calculate the optimum system $\quad$ size $\quad$ as $\quad \Omega=L / d=\sqrt{N \pi /(4(1-\varepsilon))} \quad$ since $(1-\varepsilon) \equiv V_{p} / V=N \pi d^{2} /\left(4 L^{2}\right)$ with the volume of a single particle $V_{p}$.

The pair distribution function, $g(r)$, is useful in describing short- and medium- range averaged correlations among the fibres.

\section{B.2. Nearest neighbor distance}

Nearest-neighbor distances are an essential class of spatial descriptors useful in materials science and other disciplines [36-37]. They are well established as a tool for qualitatively characterizing deviation from a "random" state. Given a set of points (fibre centers), the nearest neighbor distance distribution function for the $n$-th nearest neighbor is the probability density function $\psi_{n}(r)$ such that $\psi_{n}(r) \mathrm{d} r$ is the probability of finding the $n$-th nearest neighbor $(n=1,2,3, \ldots$, etc. $)$ in the distance range $r$ to $(r+\mathrm{d} r)$.

The analytical prediction for the mean value of the $n$-th nearest neighbor distance $\left\langle D_{n}^{p}\right\rangle$ is the first moment of the distribution function $\psi_{n}(r)$, and for the uniform random spatial distribution of points is:

$$
\left\langle D_{n}^{p}\right\rangle=\frac{\Gamma(n+1 / 2)}{\sqrt{\pi}(n-1) ! \lambda^{1 / 2}} .
$$

where $\lambda=(1-\varepsilon) / V_{p}$ is the intensity (number of points per unit area) and $\Gamma(n+1 / 2)$ is the gamma function ${ }^{4}$. While formulae have been derived that place bounds on $\left\langle D_{n}^{p}\right\rangle$ for equilibrium ensembles of monodisperse particles in two- and three-dimensions [32], exact analytical expressions are not available. Therefore, computer simulations are needed to calculate the mean value of the $n$-th nearest neighbor distances as a function of porosity (or volume fraction) for ensembles of mono(poly)disperse fibres, e.g. see Eq. (8). For more details see Section 4.1, where, among others, the mean normalized $1^{\text {st }}$ and $2^{\text {nd }}$ nearest neighbor distances are used to characterize the effective/narrow channels and predict the sample permeability.

\footnotetext{
${ }^{4}$ Note that the real unidirectional composite microstructures consist of distribution of aligned fibres of "finite" size that cannot be regarded as zero dimensional points.
} 
The $1^{\text {st }}$ nearest neighbor distance, $D_{1}^{p}$, is simply the minimum of all distances from one fibre to all others. Similar to the $g(r)$ data, at low porosities we need more fibres (bigger systems) to get reasonable statistics for the distribution of short-range distances $(N>800)$. However at high porosities, increasing the number of fibres will not much affect the probability distribution function (PDF). The distributions of $1^{\text {st }}$ nearest neighbor distances were found to follow the exponential distribution at low porosities and normal (Gaussian) distribution at high porosity. By increasing the porosity the PDF of nearest neighbor distances will change from exponential to Gaussian, i.e. a random point patterns. Furthermore, by decreasing the porosity (i.e. going from 0.9 to 0.6 ), the probability of finding a particle at exactly $1.05 d$ (i.e. minimum possible distance) becomes $\sim 10$ times larger (data not shown).

\section{B.3. Wall versus periodic boundaries}

Another factor that not only affects the fibre distribution but also the macroscopic permeability of the medium is the confining walls. In Fig. B2 the normalized permeability is plotted against number of fibres for different boundary conditions (periodic or walls at top and bottom of the cell) at (a) $\varepsilon=0.4$ and (b) $\varepsilon=0.9$. It shows that at low porosity, using the periodic boundary conditions can reduce the minimum required number of fibres $(N>200)$. However, at high porosities the permeability becomes independent of the number of fibres for $N>200$ in both periodic and wall boundary conditions. It turns out that for systems with more than 800 fibres/cylinders the effect of finite size and type of boundary conditions (periodic/no-slip/symmetric) on the permeability of the given structure diminishes.

\section{Appendix C. Towards the dense regime}

In order to have a better model for the very dense regime, we correct the original lubrication theory for perfectly hexagonal arrays, i.e. Eq. (9), in this Appendix.

As mentioned in section 3.5, the critical porosity obtained from computer simulations for the finite systems with walls is limited to $\varepsilon_{c}^{r} \cong 0.16$ [29]. By correcting the lubrication 
theory of hexagonal arrays with $\varepsilon_{c}^{r}=0.16$, one can predict the permeability at the random close packed limit more accurately as:

$$
\frac{K-K^{c}}{d^{2}}=C^{*}\left(\sqrt{\frac{1-\varepsilon_{c}^{r}}{1-\varepsilon}}-1\right)^{2.5} \text {. }
$$

where $C^{*} \sim 0.035$ is obtained by fitting to the FEM results at low porosities. Fig. C1 shows the variation of the normalized permeability as a function of porosity. Note that as we scale the data with $K^{c}$, the permeability values for different $\Delta_{\min }$, see the blue squares and the red triangles, collapse onto a single curve. As expected, Eq. (13), the proposed model based on shortest DT edges $\gamma_{1}^{T}$ (or $2^{\text {nd }}$ nearest neighbor distances, $\gamma_{2}$ ) is valid at moderate and high porosities (i.e. the range of interest in composites manufacturing, $\varepsilon^{*} \geq 0.3$ ), see the solid blue line. However, at very dense regime, i.e. $\varepsilon^{*}<0.3$, Eq. (C.1) fits better to our FE results, see the solid red line. For comparison, the analytical prediction for ordered arrays (square and hexagonal configurations), i.e. Eq. (12) is also shown with dashed lines.

Appendix D. Purely empirical, macroscopic permeability-porosity relation based on asymptotic solutions

In this appendix, based on analytical predictions of permeability for dilute and dense regimes, we present an empirical macroscopic relation for the permeability in terms of macroscopic porosity. Based on a unit cell approach, Drummond and Tahir [10] modeled analytically the flow around a fiber and obtained (applicable at high porosities):

$$
\frac{K_{D}}{d^{2}}=\frac{1}{32(1-\varepsilon)}\left(-\ln (1-\varepsilon)-1.497+2(1-\varepsilon)-\frac{(1-\varepsilon)^{2}}{2}-0.739(1-\varepsilon)^{4}+\frac{2.534(1-\varepsilon)^{5}}{1+1.2758(1-\varepsilon)}\right) \text {. }
$$

Similar to Ref. [8] and using the linear least square method, the linear correction, $g(\varepsilon)$ to the Drummond relation, leads to a corrected permeability for $\varepsilon>0.7$ as:

$$
K_{C D}=d_{1} K_{D}\left(1+d_{2} \varepsilon\right), \quad \text { with } \quad d_{1}=0.97, d_{2}=0.18
$$


with a maximum error of less than $5 \%$. Similarly, the corrected lubrication theory of hexagonal arrays, $K_{G} / d^{2}=C^{*}\left(\sqrt{\frac{1-\varepsilon_{c}^{r}}{1-\varepsilon}}-1\right)^{2.5}$ in Eq. (C.1) is valid with maximum discrepancy of less than $5 \%$ at low porosities, $\varepsilon<0.5$. To combine these two limit cases, we propose the following empirical merging function:

$$
K=K_{G}+\left(K_{C D}-K_{G}\right) p(\varepsilon) \text { with } p(\varepsilon)=\frac{1+\tanh \left(\left(\varepsilon-\varepsilon_{h}\right) / \varepsilon_{t}\right)}{2}, \varepsilon_{h} \cong 0.6, \varepsilon_{t} \cong 0.1,
$$

that is valid for the whole range of porosity, with maximum deviations of less than $5 \%$ that also includes the analytical relations for the limit cases, see the dashed blue line in Fig. 6. While the choice of $p(\varepsilon)$ is arbitrary, the nonlinear least square fitting procedure is used to obtain the empirical coefficients $\varepsilon_{h}$ and $\varepsilon_{t}$.

\section{References}

[1] R.B. Bird, W.E. Stewart and E.N. Lightfoot, Transport Phenomena, 2nd edn,. John Wiley \& Sons, 2001.

[2] K. Yazdchi, S. Srivastava, and S. Luding, Multi-Scale permeability of particulate and porous media, Proceedings of World Congress Particle Technology 6, Nürnberg Messe GmbH (Ed.), 2010, 4 pages, ISBN-978-3-00030570-2.

[3] X. Chen, T.D. Papathanasiou, The transverse permeability of disordered fiber arrays: A statistical correlation in terms of the mean nearest inter fiber spacing, Transport in Porous Media, 71 (2008), pp. 233-251.

[4] X. Chen, T.D. Papathanasiou, On the variability of the Kozeny constant for saturated flow across unidirectional disordered fibre arrays, Composites: Part A, 37 (2006), pp. 836-846.

[5] T.D. Papathanasiou, A structure-oriented micromechanical model for viscous flow through square arrays of fiber clusters, Composites Science and Technology, 56 (1996), pp. 1055-1069.

[6] Y.S. Song, K. Chung, T.J. Kang, J.R. Youn, Prediction of permeability tensor for three dimensional circular braided preform by applying a finite volume method to a unit cell, Composites Science and Technology, 64 (2004), pp. $1629-1636$. 
[7] N. Takano, M. Zako, T. Okazaki and K. Terada, Microstructure-based evaluation of the influence of woven architecture on permeability by asymptotic homogenization theory, Composites Science and Technology, 62 (2002), pp. $1347-56$

[8] K. Yazdchi, S. Srivastava and S. Luding, Microstructural effects on the permeability of periodic fibrous porous media, Int. J. Multiphase Flow, 37 (2011), pp. 956-66.

[9] D.L. Koch, J.F. Brady, The effective diffusivity of fibrous media, AIChE J., 32 (1986), pp. 575-591.

[10] J.E. Drummond and M.I. Tahir, Laminar viscous flow through regular arrays of parallel solid cylinders, Int. J. Multiphase Flow, 10 (1984), pp. 515-40.

[11] B.R. Gebart, Permeability of Unidirectional Reinforcements for RTM, Journal of Composite Materials, 26 (1992), pp. 1100-33.

[12] P.C. Carman, Fluid flow through granular beds, Transactions of the Institute of Chemical Engineering, 15 (1937), pp. 150-66.

[13] T.G. Gutowski, T. Morigaki and Z. Cai, The Consolidation of Laminate Composites, Journal of Composite Materials, 21 (1987), pp. 172-188.

[14] M.V. Bruschke and S.G. Advani, Flow of generalized Newtonian fluids across a periodic array of cylinders, Journal of Rheology, 37 (1993), pp. 479-98.

[15] B. Astroem, R. Pipes, S. Advani, On flow through aligned fiber beds and its application to composite processing, Journal of Composite Materials, 26 (1992), pp. 1351-73.

[16] A.J. Katz, A.H. Thompson, Quantitative prediction of permeability in porous rocks, Phys. Rev. B, 34 (1986), pp. 8179-81.

[17] K. Yazdchi, S. Srivastava and S. Luding, On the transition from creeping to inertial flow in arrays of cylinders, Proceedings of IMECE, 2010, 6 pages, ISBN-978-0-7918-3891-4, Vancouver, British Columbia, Canada.

[18] D.L. Koch, A.J.C. Ladd, Moderate Reynolds number flows through periodic and random arrays of aligned cylinders, J. Fluid Mech., 349 (1997), pp. 31-66.

[19] R.J. Hill, D.L. Koch and A. J. C. Ladd, Moderate-Reynolds-number flows in ordered and random arrays of spheres, J. Fluid Mech., 448 (2001), pp. 243-78.

[20] R.J. Hill, D.L. Koch and A. J. C. Ladd, The first effects of fluid inertia on flows in ordered and random arrays of spheres, J. Fluid Mech., 448 (2001), pp. 21341. 
[21] R. Dickman, J.S. Wang and I. Jensen, Random sequential adsorption: Series and virial expansions, J. Chem. Phys., 94 (1991), pp. 8252-58.

[22] E. L. Hinrichsen, J. Feder and T. Jøssang, Geometry of random sequential adsorption, J. Stat. Phys., 44 (1986), pp. 793-827.

[23] X. Chen, T.D. Papathanasiou, Micro-scale modeling of axial flow through unidirectional disordered fiber arrays, Composites Science and Technology, 67 (2007), pp. 1286-1293.

[24] K.W. Desmond and E.R. Weeks, Random close packing of disks and spheres in confined geometries, PRE, 80 (2009), 051305.

[25] S. Luding, Cohesive frictional powders: Contact models for tension, Granular Matter, 10 (2008), pp. 235-246.

[26] R. Pyrz, Quantitative description of the microstructure of composites. Part I: morphology of unidirectional composite systems, Composites Science and Technology, 50 (1994), pp. 197-208.

[27] I. Schenker, F.T. Filser, L.J. Gauckler, T. Aste and Hans J. Herrmann, Quantification of the heterogeneity of particle packings, PRE 80 (2009), 021302 .

[28] A. S. Sangani and C. Yao, Transport processes in random arrays of cylinders. II. Viscous flow, Phys. Fluids 31 (1988), pp. 2435-44.

[29] James G. Berryman, Random close packing of hard spheres and disks, Phys. Rev. A 27 (1983), pp. 1053-1061.

[30] A. Okabe, B. Boots and K. Sugihara, Spatial Tessellations: Concepts and Applications of Voronoi Diagrams, Wiley, Chichester, UK, 1992.

[31] Ascânio D. Araújo, Wagner B. Bastos, José S. Andrade, Jr., and Hans J. Herrmann, Distribution of local fluxes in diluted porous media, PRE 74 (2006), 010401.

[32] O. Duran, N.P. Kruyt and S. Luding, Analysis of three-dimensional micromechanical strain formulations for granular materials: evaluation of accuracy, Int. J. of Solids and Structures 47 (2010), pp. 251-260.

[33] C. Grufman, F. Ellyin, Determining a representative volume element capturing the morphology of fibre reinforced polymer composites, Composites Science and Technology, 67 (2007), pp. 766-775.

[34] X. Du and M. Ostoja-Starzewski, On the size of representative volume element for Darcy law in random media, Proc. R. Soc. A, 462 (2006), pp. 2949-2963. 
[35] D. Trias , J. Costa, A. Turon, J.E. Hurtado, Determination of the critical size of a statistical representative volume element (SRVE) for carbon reinforced polymers, Acta Materialia, 54 (2006), pp. 3471-3484.

[36] A.R. Melro, P.P. Camanho, S.T. Pinho, Generation of random distribution of fibres in long-fibre reinforced composites, Composites Science and Technology 68 (2008), pp. 2092-2102.

[37] S. Torquato, Random heterogeneous materials - microstructure and macroscopic properties. Springer-Verlag, New York, 2002. 
Table 1: The values of the critical porosity, $\varepsilon_{\Delta_{\min }}^{\text {hex }}=1-\left(1-\varepsilon_{\text {hex }}^{*}\right) /\left(1+\Delta_{\min }\right)^{2}$ with $\varepsilon_{\text {hex }}^{*}=1-\pi /(2 \sqrt{3}) \cong 0.0931$, and permeability (for a perfectly hexagonal lattice, $K_{\Delta_{\min }}^{\text {hex }} / d^{2}$, and corrected permeability for random lattices $K^{\mathrm{c}} / d^{2}$ ) at different $\Delta_{\text {min }}$.

\begin{tabular}{|c|c|c|c|}
\hline$\Delta_{\min }$ & $\varepsilon_{\Delta_{\min }}^{\mathrm{hex}}$ & $K_{\Delta_{\min }}^{\text {hex }} / d^{2}$ & $K^{\mathrm{c}} / d^{2}$ \\
\hline 0.2 & 0.3702 & $1.035 \times 10^{-3}$ & $1.7 \times 10^{-3}$ \\
\hline 0.1 & 0.2505 & $1.801 \times 10^{-4}$ & $4.3 \times 10^{-4}$ \\
\hline 0.05 & 0.1774 & $3.172 \times 10^{-5}$ & $1.3 \times 10^{-4}$ \\
\hline 0.025 & 0.1368 & $5.587 \times 10^{-6}$ & $3.5 \times 10^{-5}$ \\
\hline 0.005 & 0.1021 & $1.006 \times 10^{-7}$ & $2.8 \times 10^{-6}$ \\
\hline
\end{tabular}

Table 2: The numerical values of $\varepsilon_{c}^{r}=1-\left(1-\varepsilon_{r}^{*}\right) /\left(1+\Delta_{\text {min }}\right)^{2}$ with $\varepsilon_{r}^{*} \cong 0.16,\left\langle D_{n}^{p}\right\rangle$, $\varphi_{n}$ and $\xi_{n}$ for various $n$ and $\Delta_{\min }$ which are obtained analytically from Eq. (B.1) and by least square fitting of numerical simulation in the range of $0.3<\varepsilon^{*}<0.95$.

\begin{tabular}{|c|c|c|c|c|c|}
\hline \multirow{3}{*}{$\Delta_{\min }$} & $n$ & $\varepsilon_{c}^{r}$ & $\left\langle D_{n}^{p}\right\rangle$ & $\varphi_{n}$ & $\xi_{n}$ \\
\hline \multirow{3}{*}{0.2} & 1 & 0.4167 & 0.3535 & 1.0727 & 0.7904 \\
\cline { 2 - 6 } & 2 & 0.4167 & 0.5303 & 0.3372 & 0.6790 \\
\cline { 2 - 6 } 0.1 & 3 & 0.4167 & 0.6629 & 0.1049 & 0.2502 \\
\cline { 2 - 6 } & 1 & 0.3058 & 0.3535 & 1.0757 & 0.7910 \\
\cline { 2 - 6 } & 3 & 0.3058 & 0.5303 & 0.3509 & 0.7048 \\
\hline \multirow{3}{*}{0.05} & 1 & 0.2381 & 0.3535 & 1.0732 & 0.2454 \\
\cline { 2 - 6 } & 2 & 0.2381 & 0.5303 & 0.3495 & 0.7867 \\
\cline { 2 - 6 } & 3 & 0.2381 & 0.6629 & 0.1064 & 0.7017 \\
\hline \multirow{3}{*}{0.025} & 1 & 0.2005 & 0.3535 & 1.0771 & 0.7887 \\
\cline { 2 - 6 } & 2 & 0.2005 & 0.5303 & 0.3557 & 0.7084 \\
\cline { 2 - 6 } & 3 & 0.2005 & 0.6629 & 0.1099 & 0.2567 \\
\hline \multirow{3}{*}{0.005} & 1 & 0.1683 & 0.3535 & 1.0806 & 0.7948 \\
\cline { 2 - 6 } & 2 & 0.1683 & 0.5303 & 0.3611 & 0.7314 \\
\cline { 2 - 6 } & 3 & 0.1683 & 0.6629 & 0.1123 & 0.2969 \\
\hline
\end{tabular}




\section{Figure captions:}

Fig. 1: Fibre distributions generated by a Monte Carlo procedure (see subsection 3.2), with 200 unidirectional cylinders, normal (y) to the flow direction (x), with minimum inter fibre distance $\Delta_{\min }=0.05$ at porosity $\varepsilon=0.6$. At the top the diameter, $d$ and virtual diameter $d^{*}=d\left(1+\Delta_{\text {min }}\right)$ are shown, schematically. At the top 3D and bottom 2D representation of fibre distribution are shown. The zoom shows the fine, unstructured, triangular FEM mesh.

Fig. 2: (a) plots of $g(r)$ for 800 fibres generated by different methods with $\Delta_{\min }=0.05$ at porosity $\varepsilon=0.6$, (b) comparison of the normalized permeability of the fibre arrangements from (a) plotted against the number of fibres. All data are averaged over 10 realizations with $10^{4}$ perturbations. The error bars indicate the standard deviation. Larger numbers of perturbations do not lead to a visible difference.

Fig. 3: Each image consists of 800 fibres with minimum inter fibre distance $\Delta_{\text {min }}=0.05$ and $\varepsilon=0.6$. They are generated by (a) Random Placement (RP), (b) Monte Carlo (MC) procedure, (c) Energy Minimization (EM) approach and (d) Molecular Dynamics (MD) simulations. The red box shows the center area which has been used to calculate $g(r)$. For the chosen reference particles only those in the inner red square are used, while the distances to all others are considered.

Fig. 4: Normalized permeability as a function of porosity for homogenous (generated with MC procedure or MD simulations) and clustered correlated (generated with EM approach) structure in both horizontal and vertical directions. Error bars indicate standard deviation from 10 realizations.

Fig. 5: Typical fibre distributions generated by a Monte Carlo (MC) procedure, each with 800 fibres at $\varepsilon=0.5$ with minimum inter fibre distance (a) $\Delta_{\min }=0.2\left(\varepsilon^{*}=0.28\right)$ and (b) $\Delta_{\min }=0.005\left(\varepsilon^{*}=0.49\right)$. The color code shows the horizontal velocity field in a pressure driven system. Only the center part of the system is shown.

Fig. 6: Variation of corrected normalized permeability plotted against porosity for different minimum inter fibre distances $\Delta_{\min }$, with $N=800$. The five arrows show the expected onset of ordering at $\varepsilon_{\text {order }}^{*} \approx 0.3$ for decreasing $\Delta_{\min }$ (from right to left). The dashed blue line shows the empirical merging function, Eq. (D.3), in appendix D. The inset shows the low permeability data without scaling at low porosities. The dashed red line and blue stars correspond to the periodic hexagonal cell values of lubrication theory [11] and finite element results [8], respectively.

Fig. 7: Various microstructural descriptors used in this study: (a) The $2^{\text {nd }}$ and (b) the $1^{\text {st }}$ nearest neighbor distances plotted for each fibre. (c) The blue lines delineate the Delaunay triangles. (d) The minimum Delaunay edges plotted for each DT. The red lines show the repeated edges from neighboring triangles. All graphs show the center part of 
800 randomly distributed fibres generated by the MC procedure at $\varepsilon=0.6$ with minimum inter fibre distance $\Delta_{\min }=0.05$.

Fig. 8: Variation of normalized mean nearest neighbor distances $\left(1^{\text {st }}, 2^{\text {nd }}\right.$ and $\left.3^{\text {rd }}\right)$, the mean length of all Delaunay edges $\left(\gamma_{D T}^{p}\right)$, the mean shortest Delaunay edge averaged over particles $\left(\gamma_{1}^{p}\right)$ and triangles $\left(\gamma_{1}^{T}\right)$ and the normalized hydraulic diameter $\left(D_{h} / d\right)$ as a function of porosity. Average is taken over 10 realizations with 800 randomly distributed fibres. Only the center part of the system is considered to avoid edge effects.

Fig. 9: Variation of mean values of the $2^{\text {nd }}$ nearest neighbors, $\gamma_{2}$ as function of porosity together with the best fits from Eq. (8) (solid lines) at different $\Delta_{\min }$. The inset shows the scaled data by plotting $\gamma_{2}^{*}$ as function of effective porosity $\varepsilon^{*}$.

Fig. 10: Variation of normalized permeability, plotted as function of various statistical descriptors. $2^{\text {nd }}$ nearest neighbor distance $\gamma_{2}$ (or shortest Delaunay edges, $\gamma_{1}^{T}$ ) show the best (almost power law) correlation in a wide range of porosity at $\Delta_{\min }=0.05$. The solid blue line shows the power law fit.

Fig. 11: Variation of normalized permeability as function of mean value of $2^{\text {nd }}$ nearest neighbor distance, $\gamma_{2}$ at different values of $\Delta_{\min }$.

Fig. A1: Plot of normalized permeability for different resolutions (number of elements, $\mathrm{N}_{\mathrm{e}}$ ) at porosity $\varepsilon=0.4$ (in blue) and $\varepsilon=0.8$ (in red).

Fig. B1: Plot of $g(r)$ for different number of fibres (system size) at (a) porosity $\varepsilon=0.4$ and (b) $\varepsilon=0.9$ from 10 realizations. The dashed line at $g(r)=1$ indicates a completely random point structure. The drop of the data comes from the finite size of the center area used for averaging (see Fig. 3).

Fig. B2: Variation of normalized permeability versus number of fibres with different boundary conditions at (a) porosity $\varepsilon=0.4$ and (b) $\varepsilon=0.9$. Fibre distributions generated by MC procedure with $10^{4}$ perturbations and minimum inter fibre distance $\Delta_{\min }=0.05$.

Fig. C1: Variation of scaled normalized permeability plotted against porosity. 

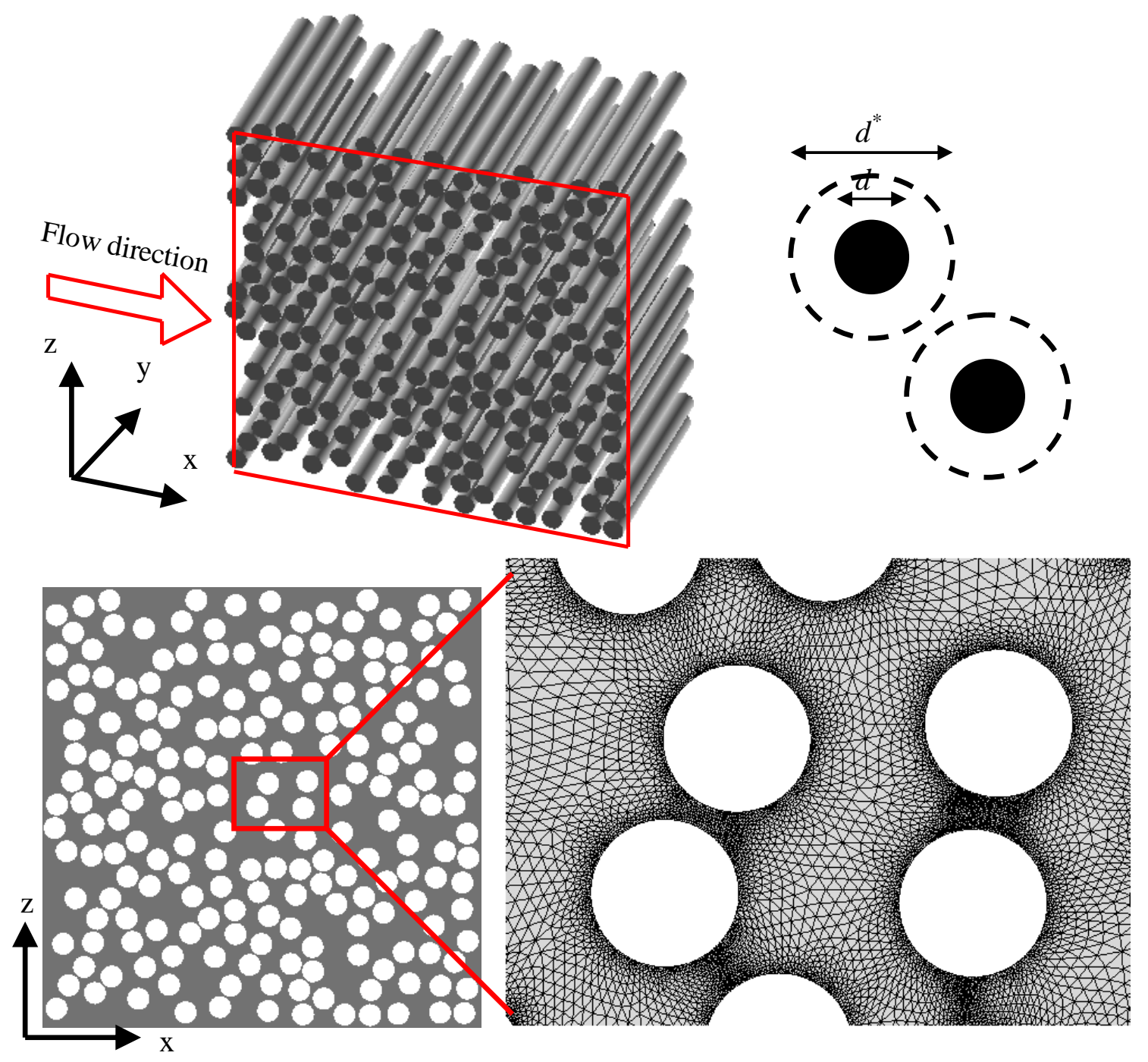

Figure 1. 

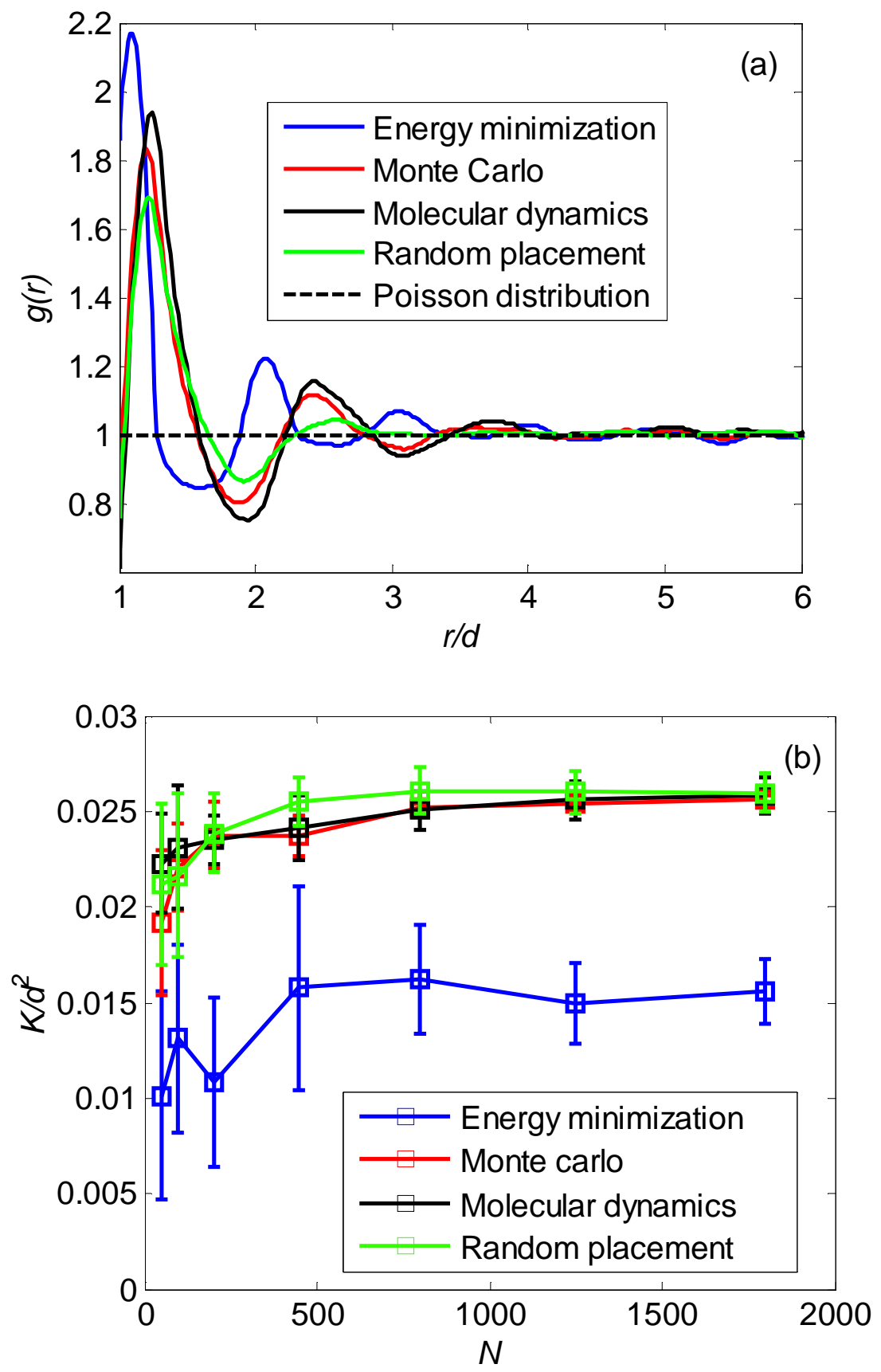

Figure 2. 


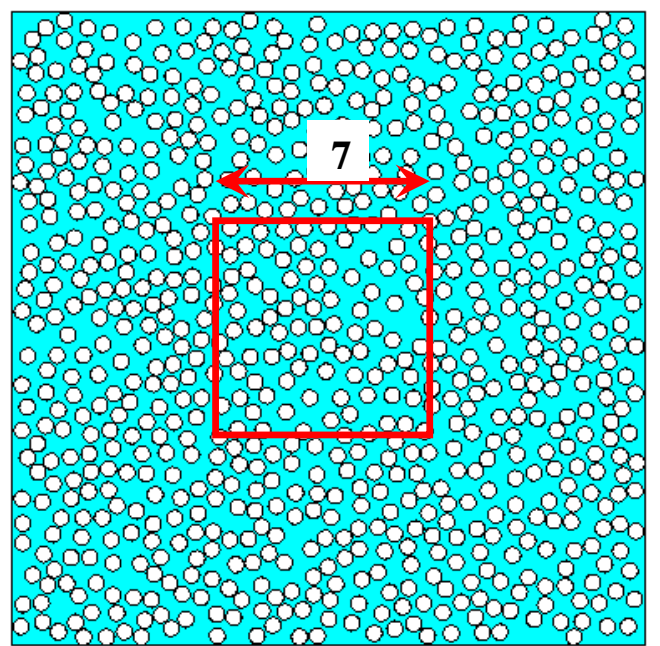

(a)

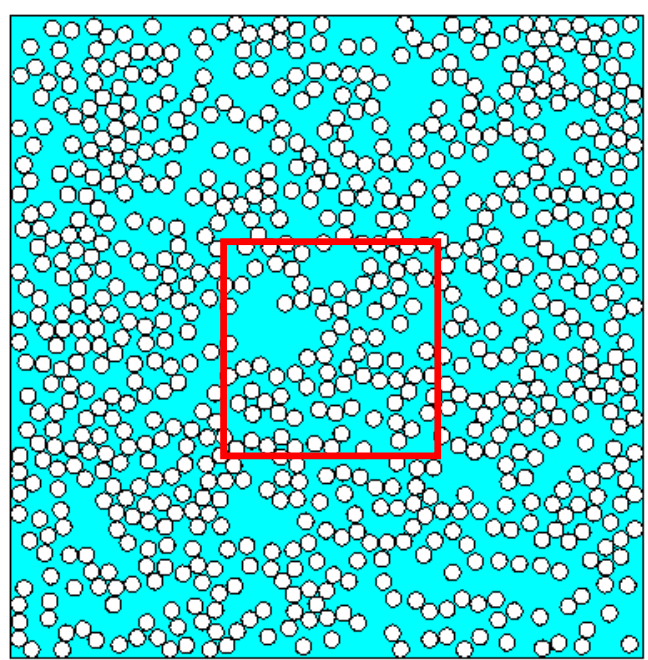

(c)

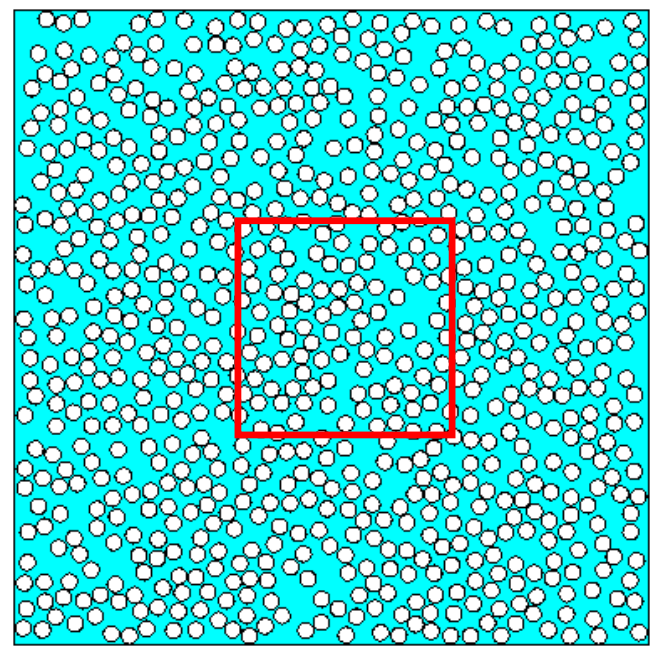

(b)

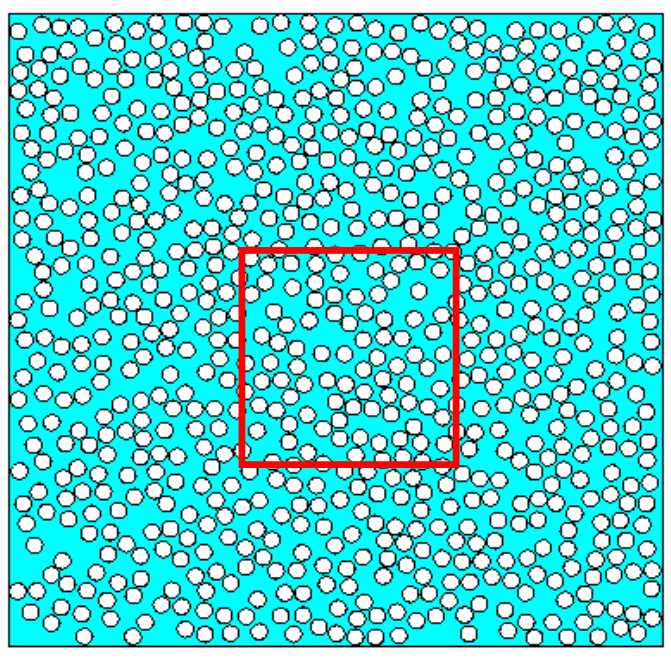

(d)

Figure 3. 


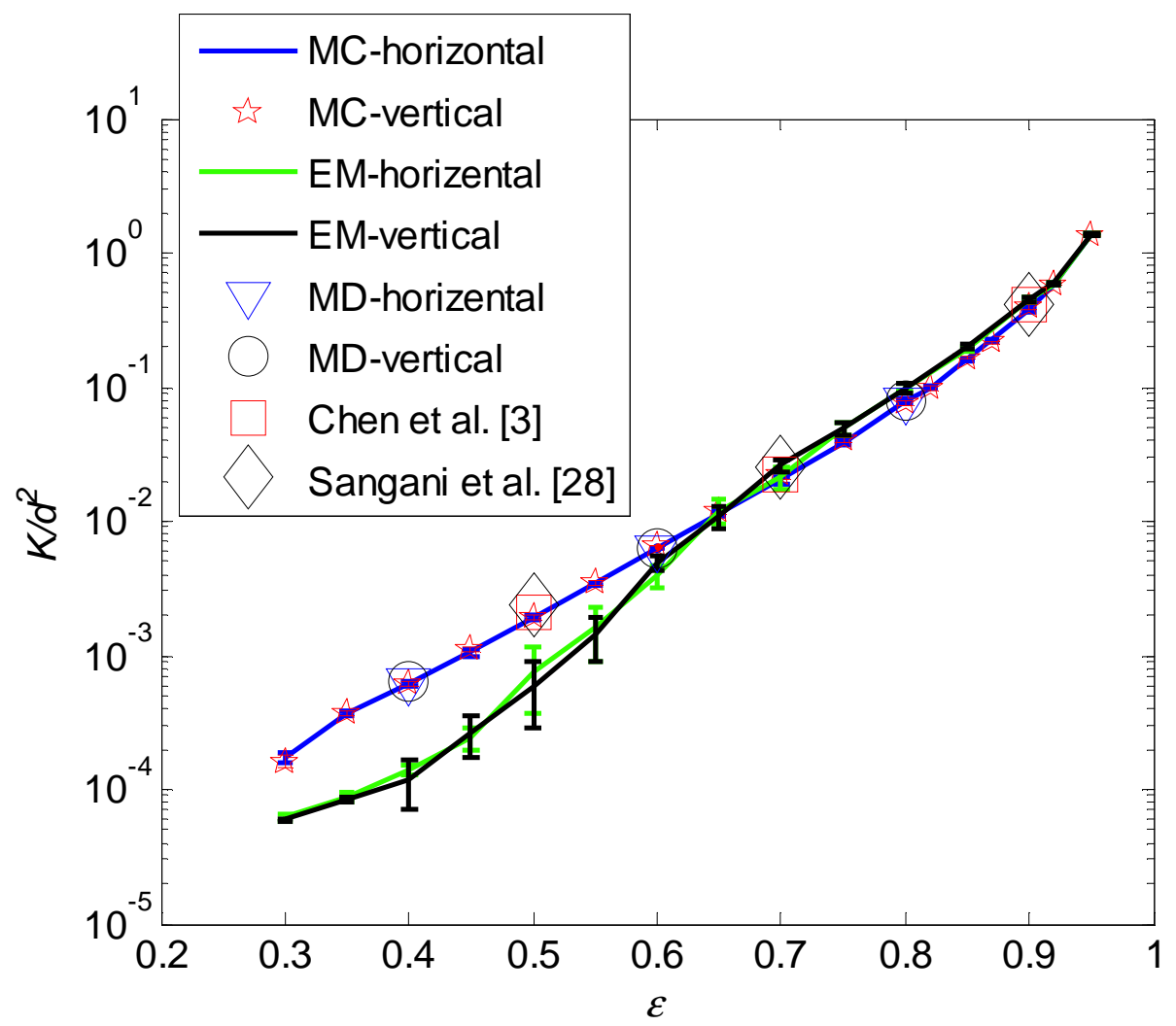

Figure 4.
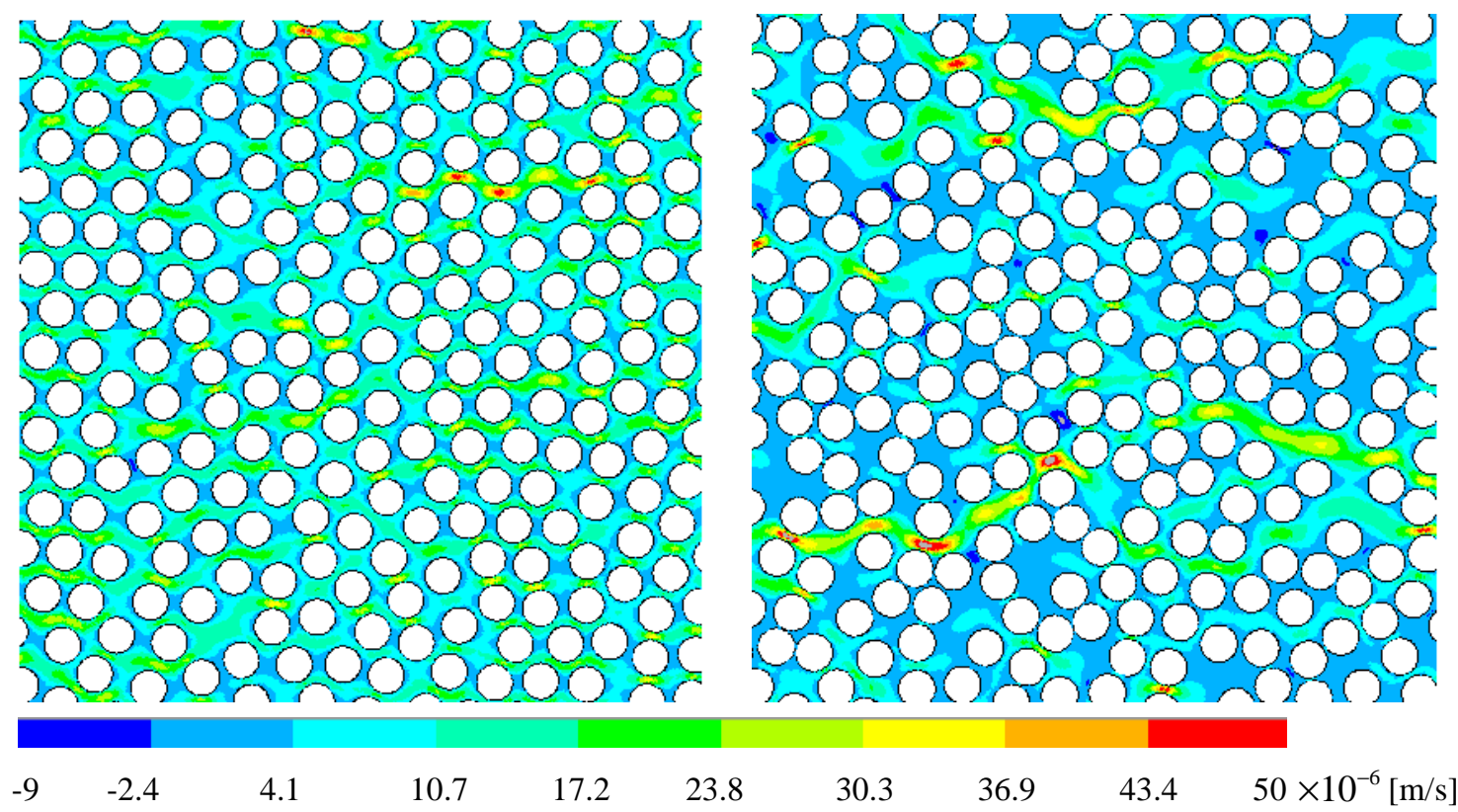

$\begin{array}{llll}-9 & -2.4 & 4.1 & \\ & & & \end{array}$

$\begin{array}{lll}10.7 & 17.2 & 23.8\end{array}$

30.3

(b)

Figure 5. 


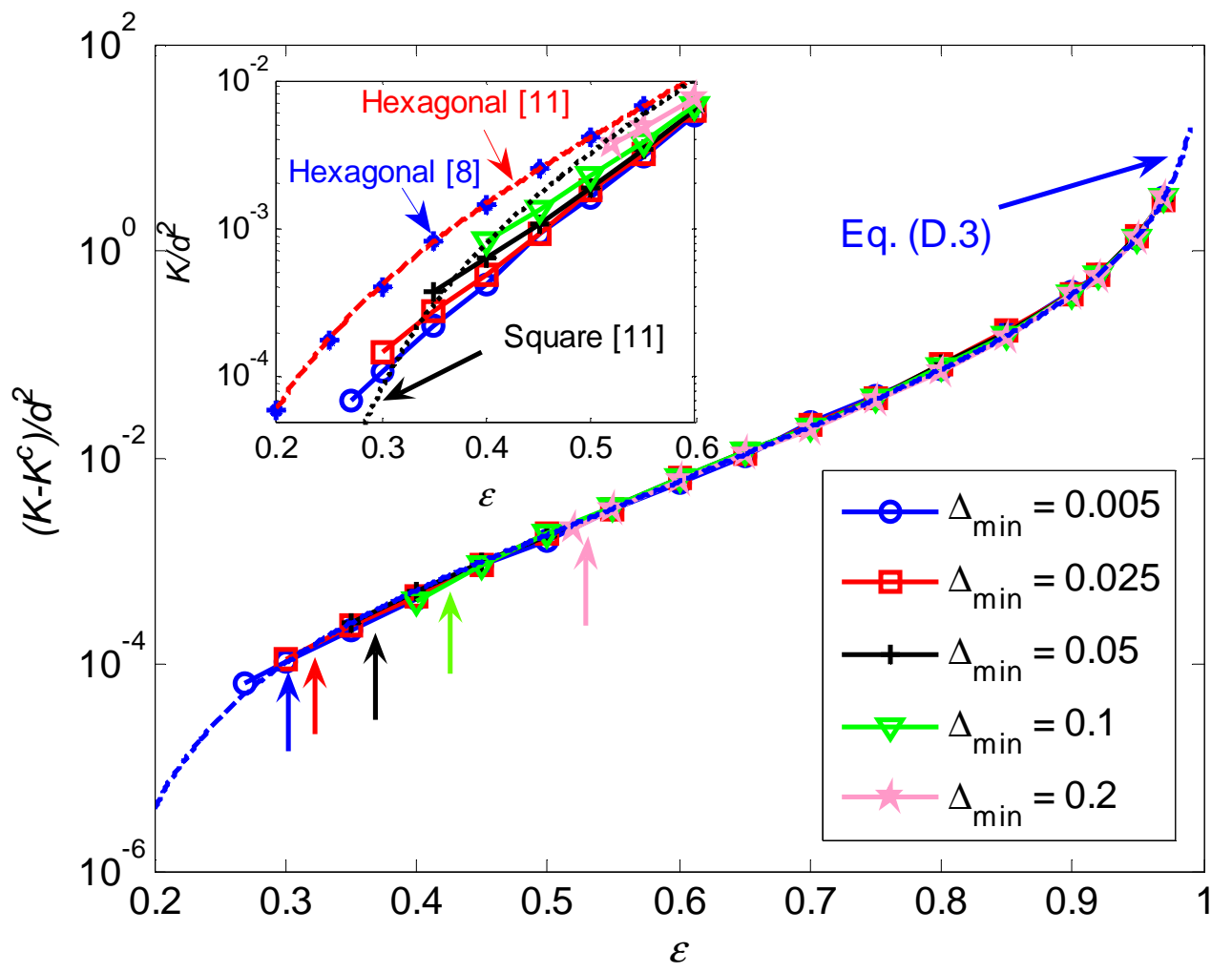

Figure 6. 


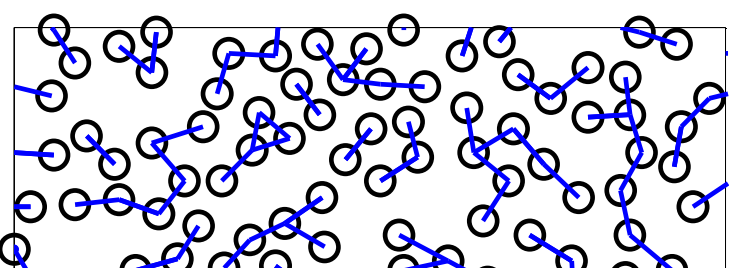

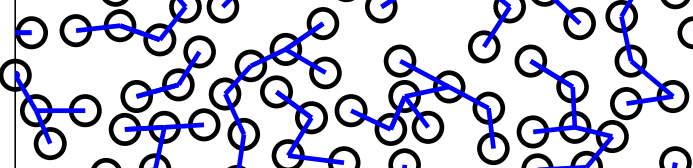
OO $\$ 00$ Q 00 \%O IQ $\$ 9 \$ 0^{\circ}$ - 00.00080

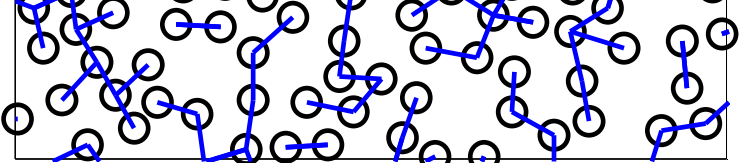
(a)

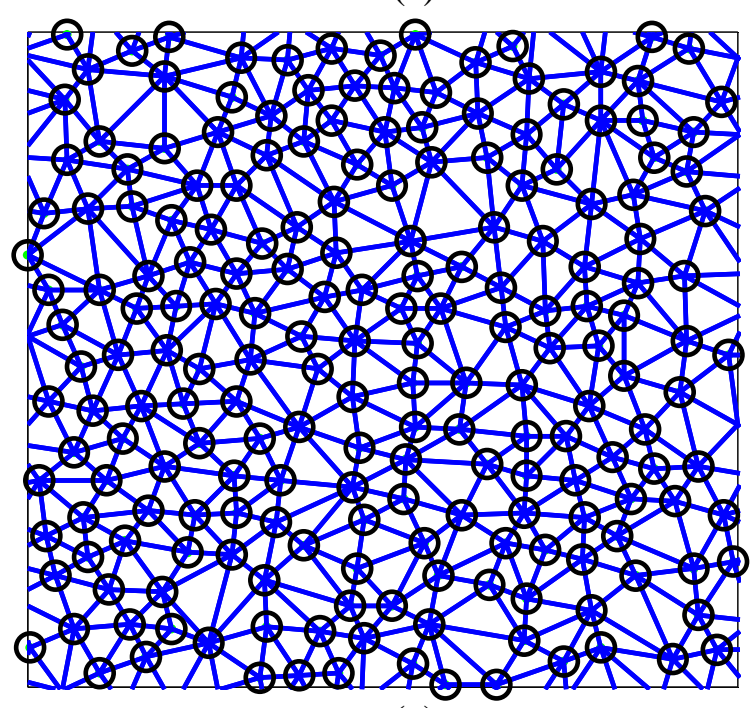

(c)
0008008000 0000000000 $0000 \% 8000 \%$ $0000 \% 80 \%$ - Q.0 o 0000 $00000 \$ 000$ 080.000000000

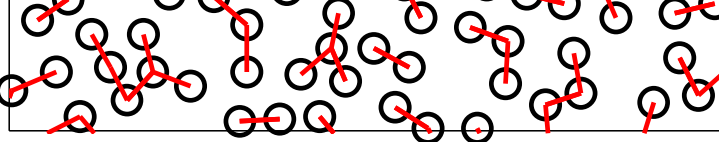

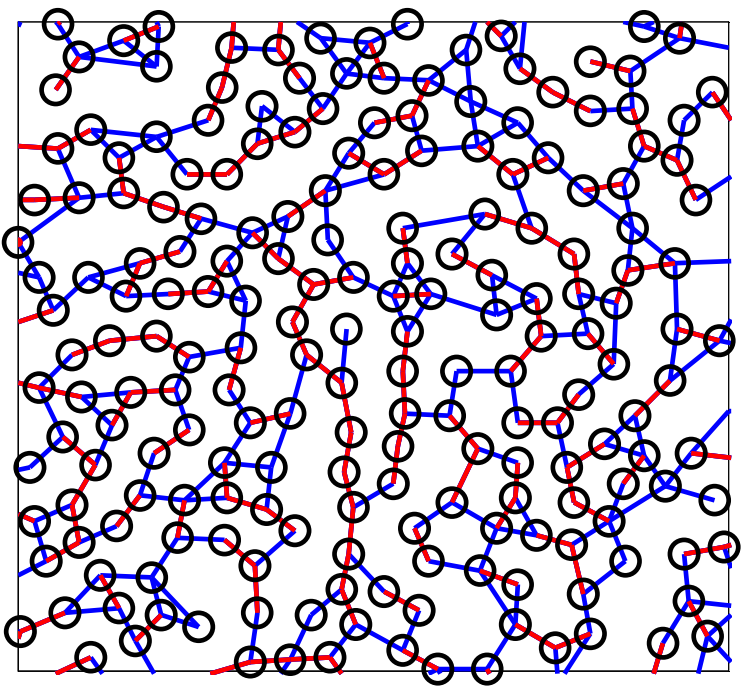

(d)

Figure 7. 


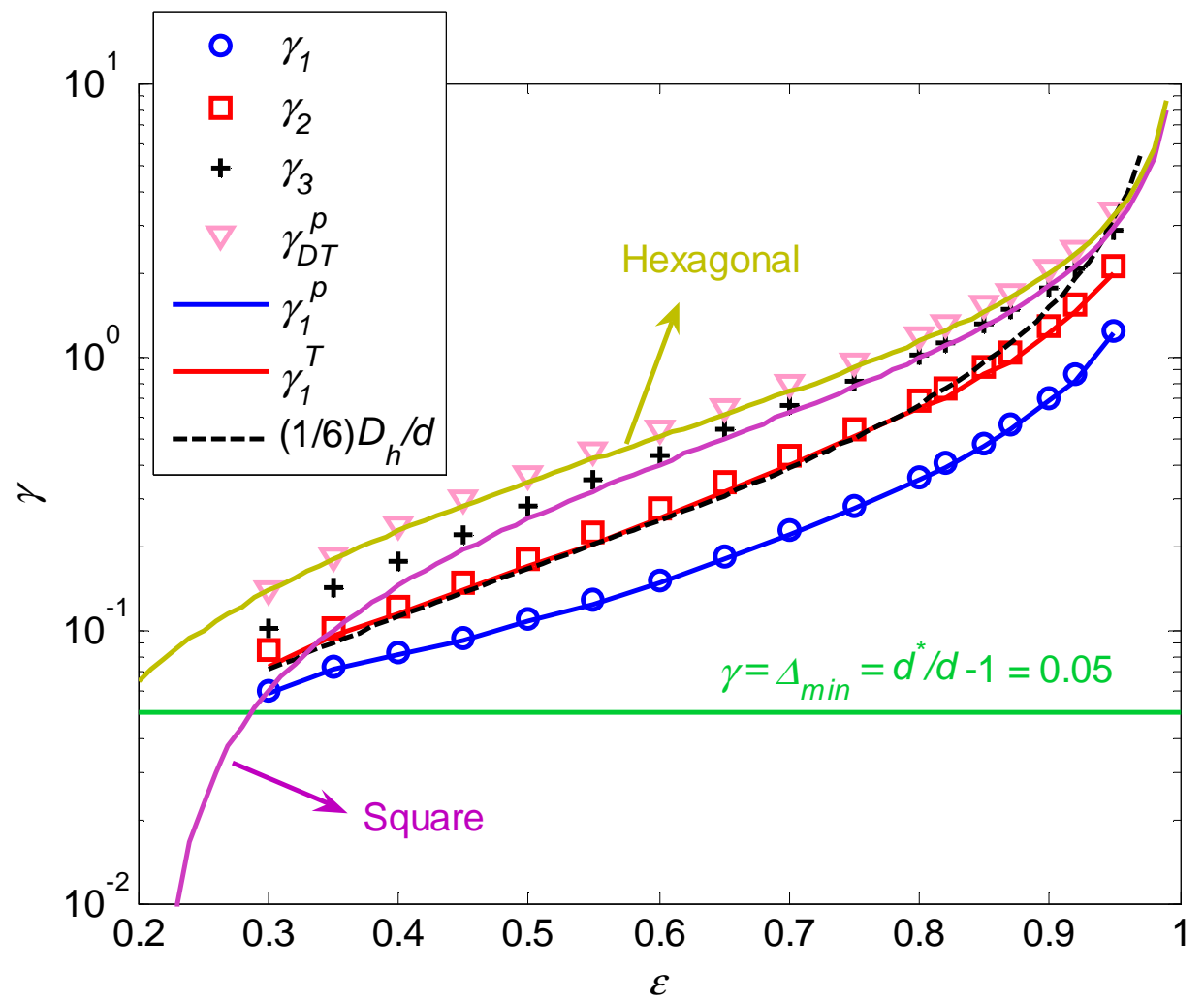

Figure 8.

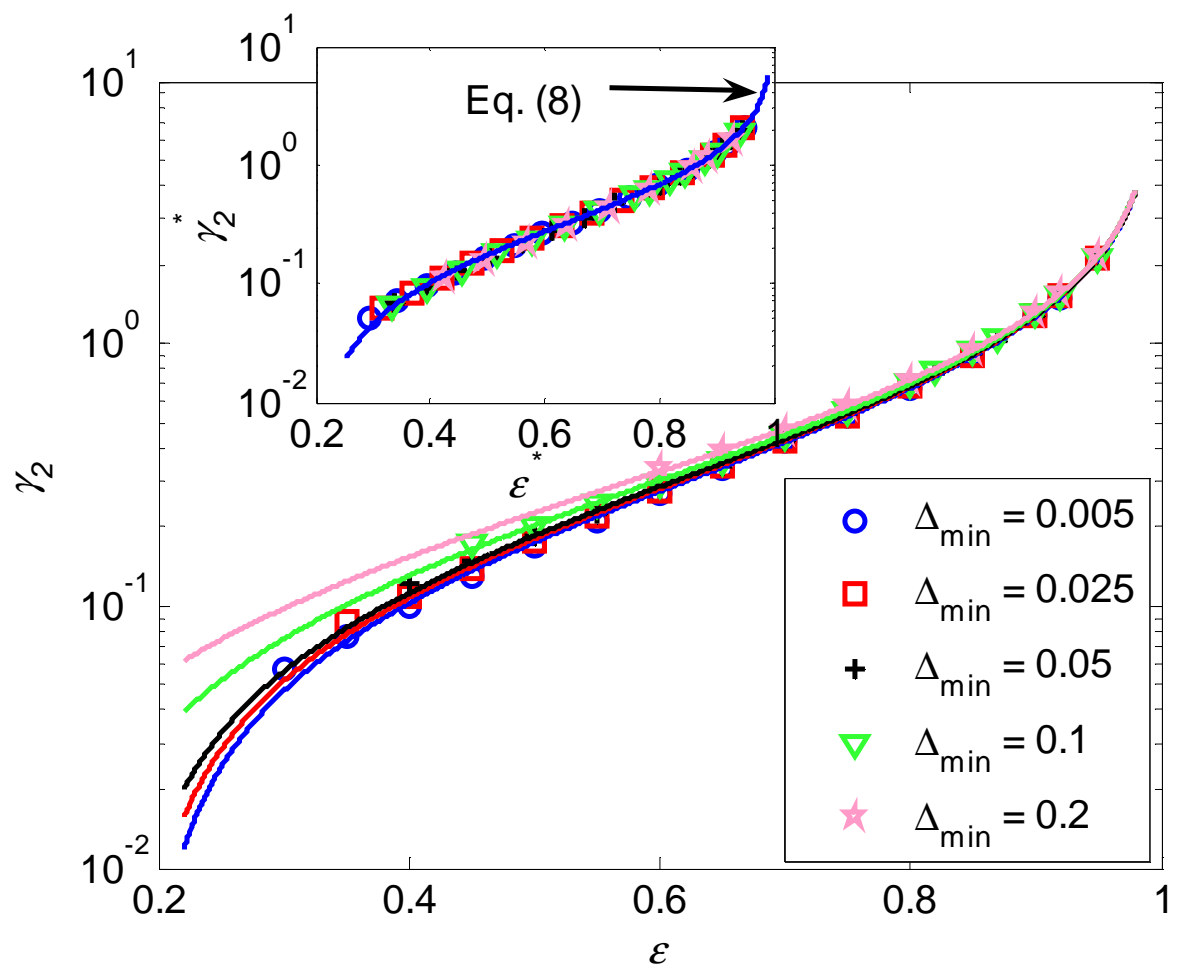

Figure. 9 


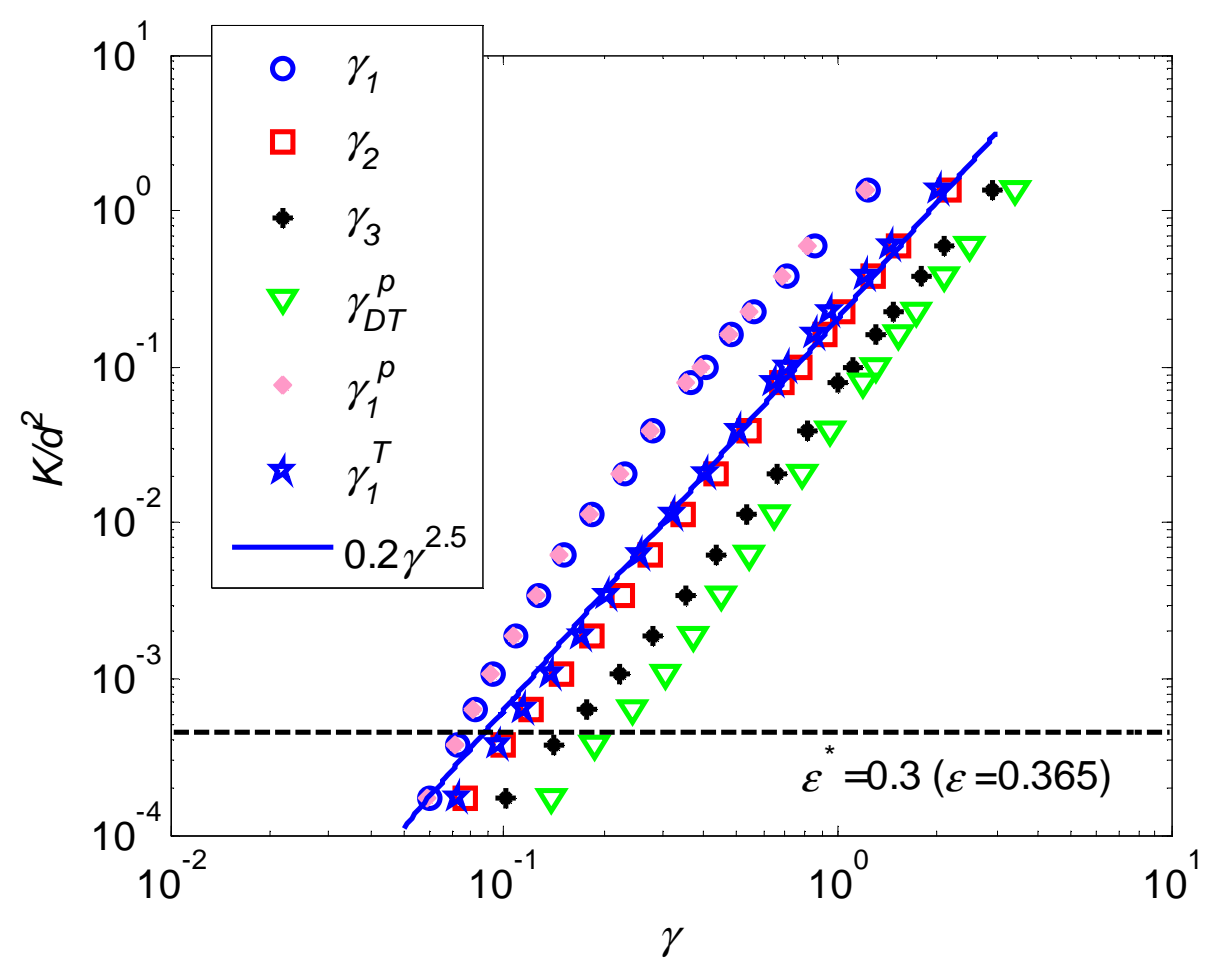

Figure 10.

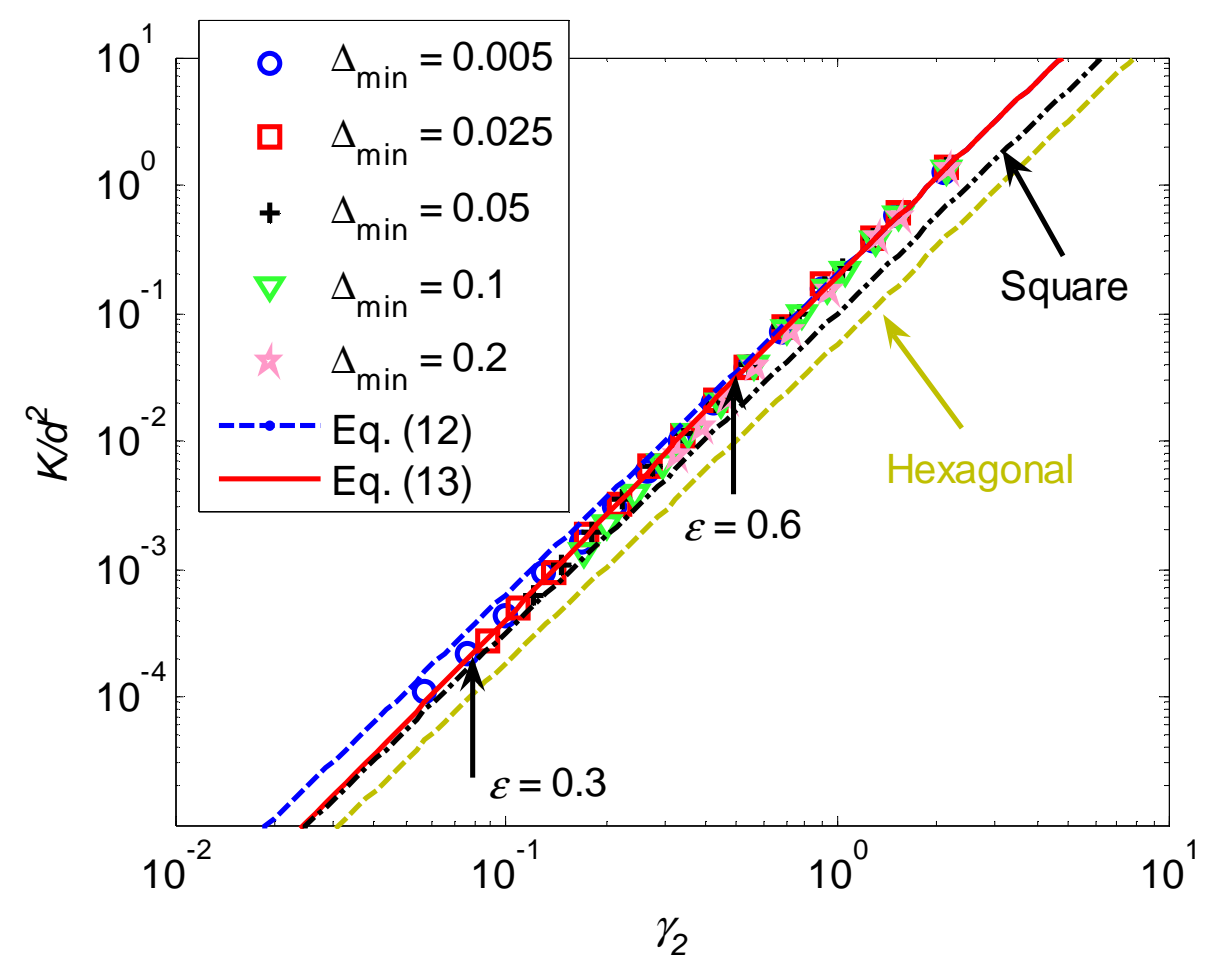

Figure 11. 


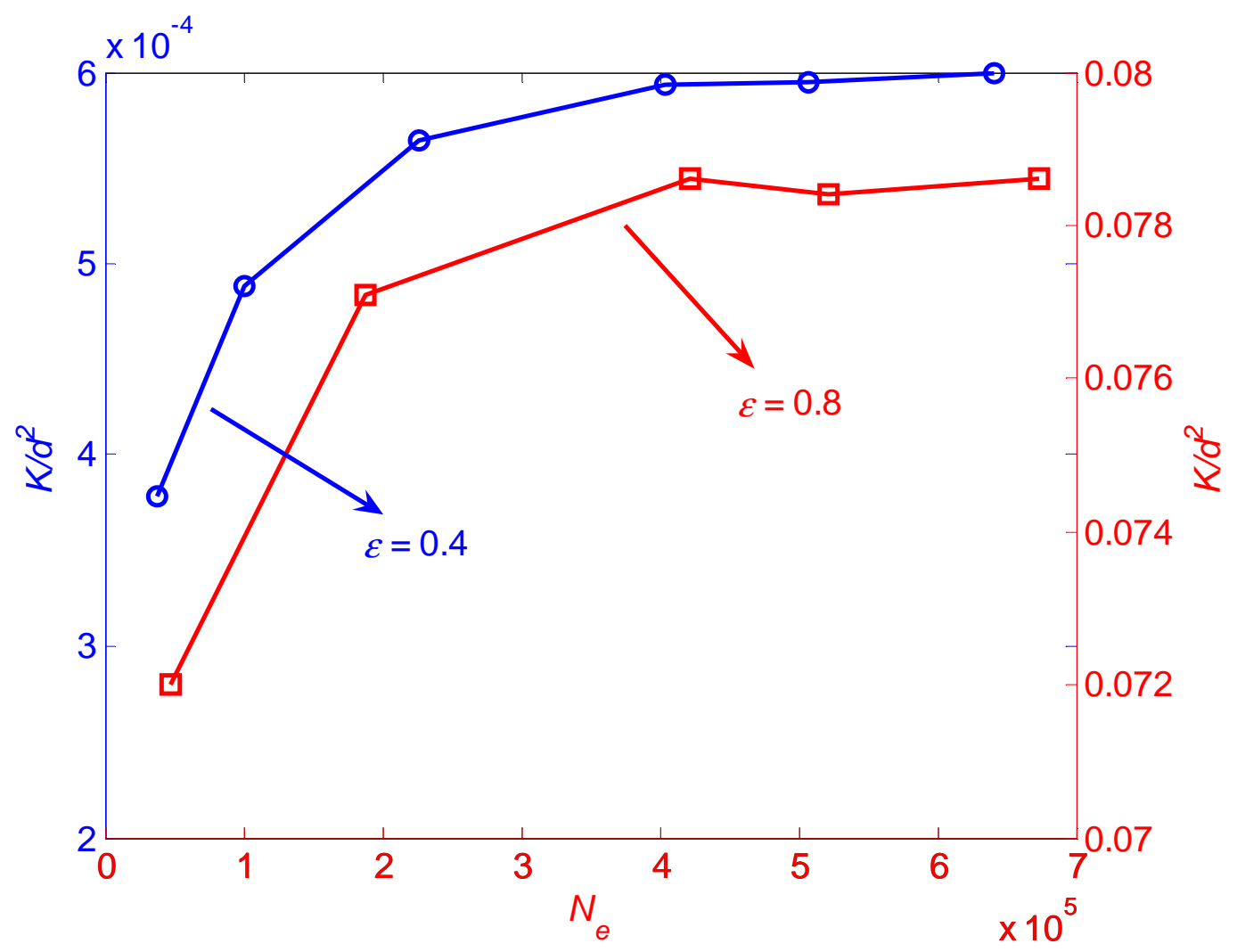

Figure A1.

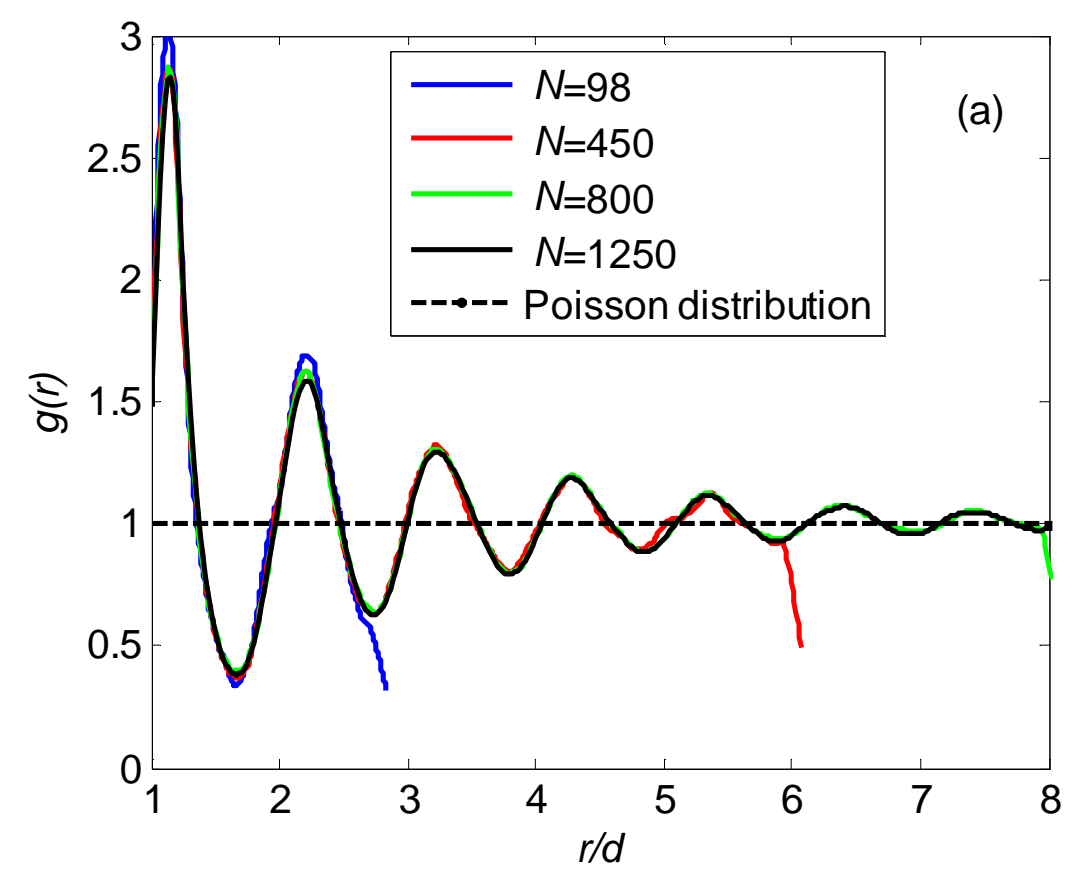




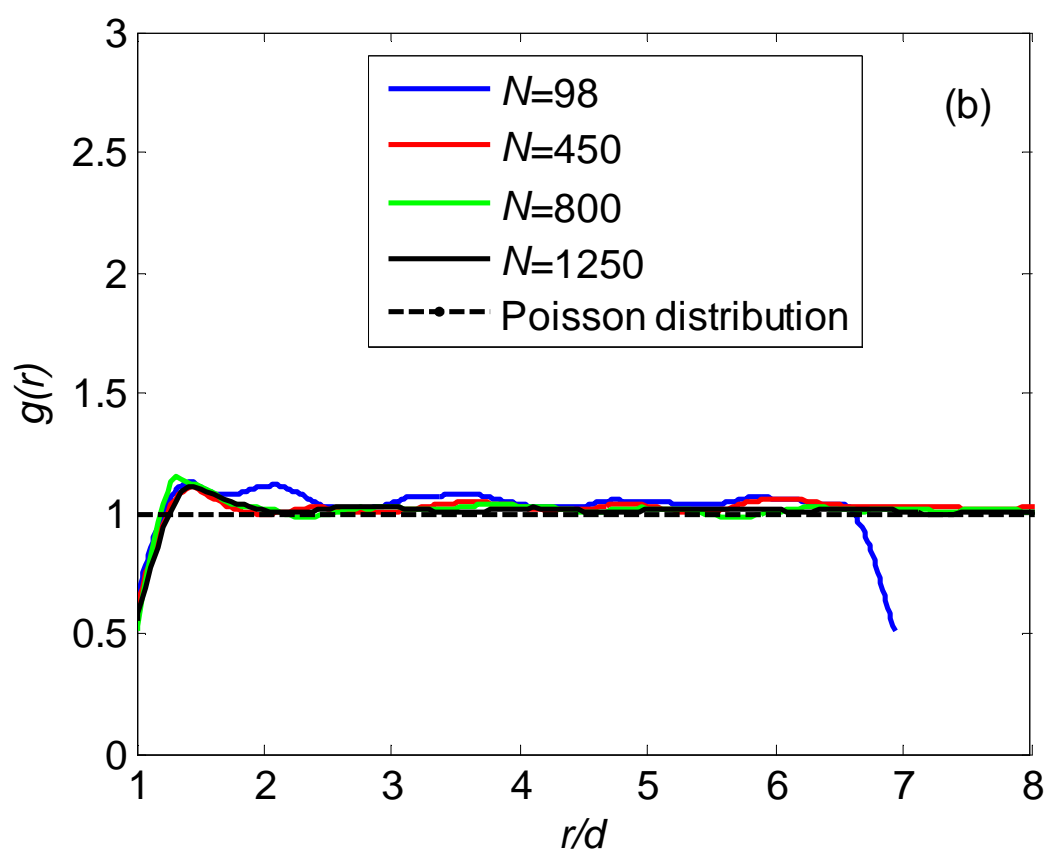

Figure B1.

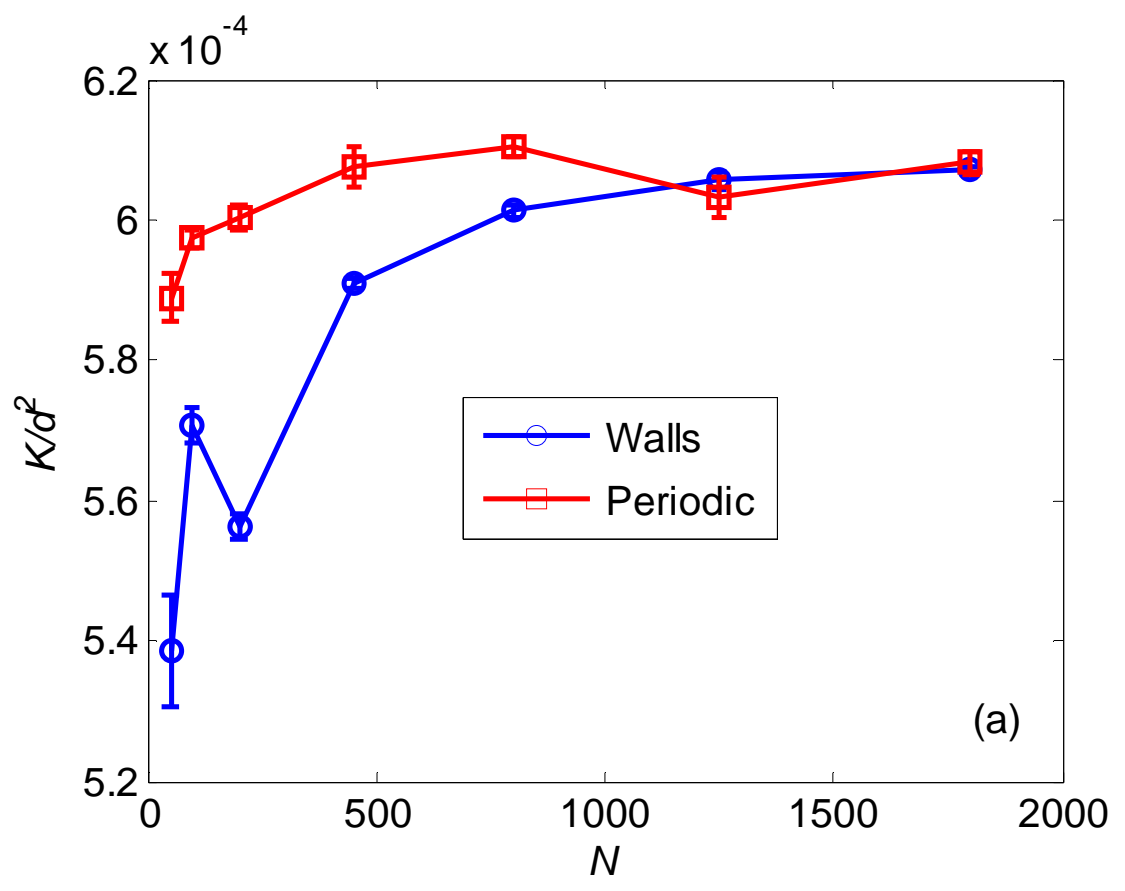




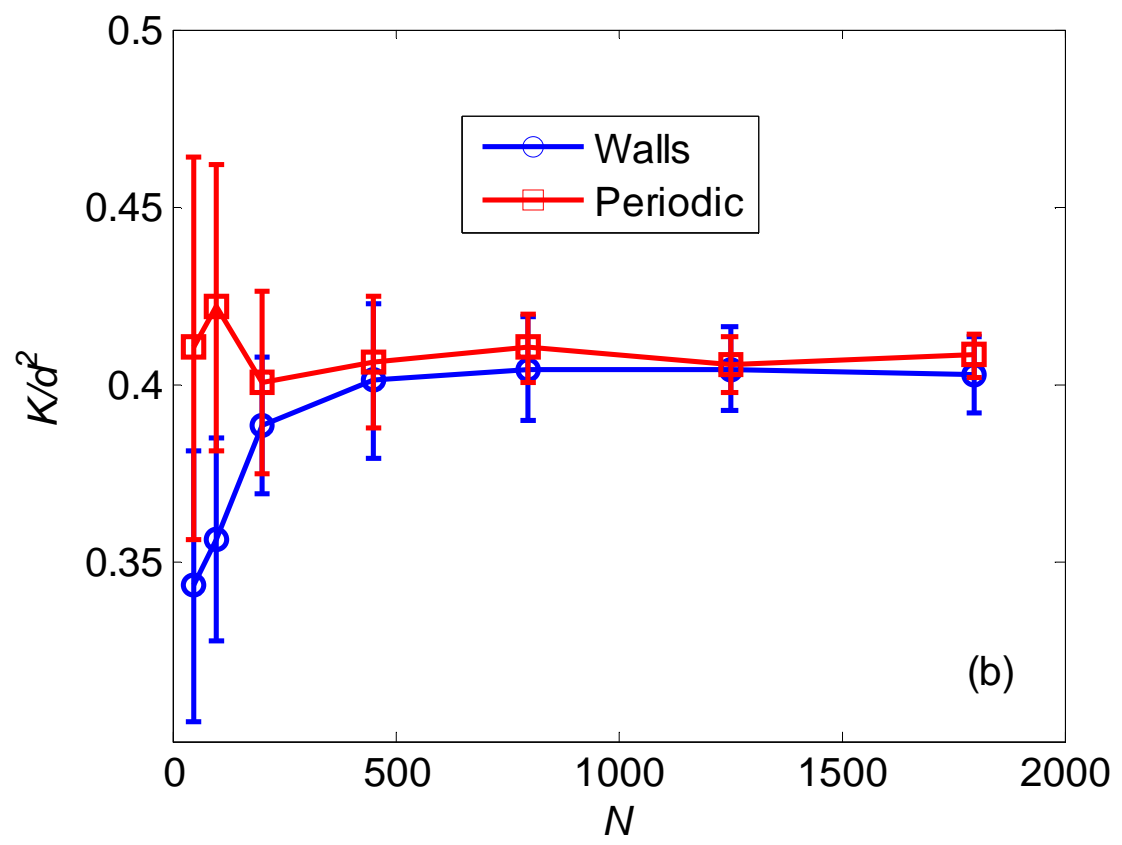

Figure B2.

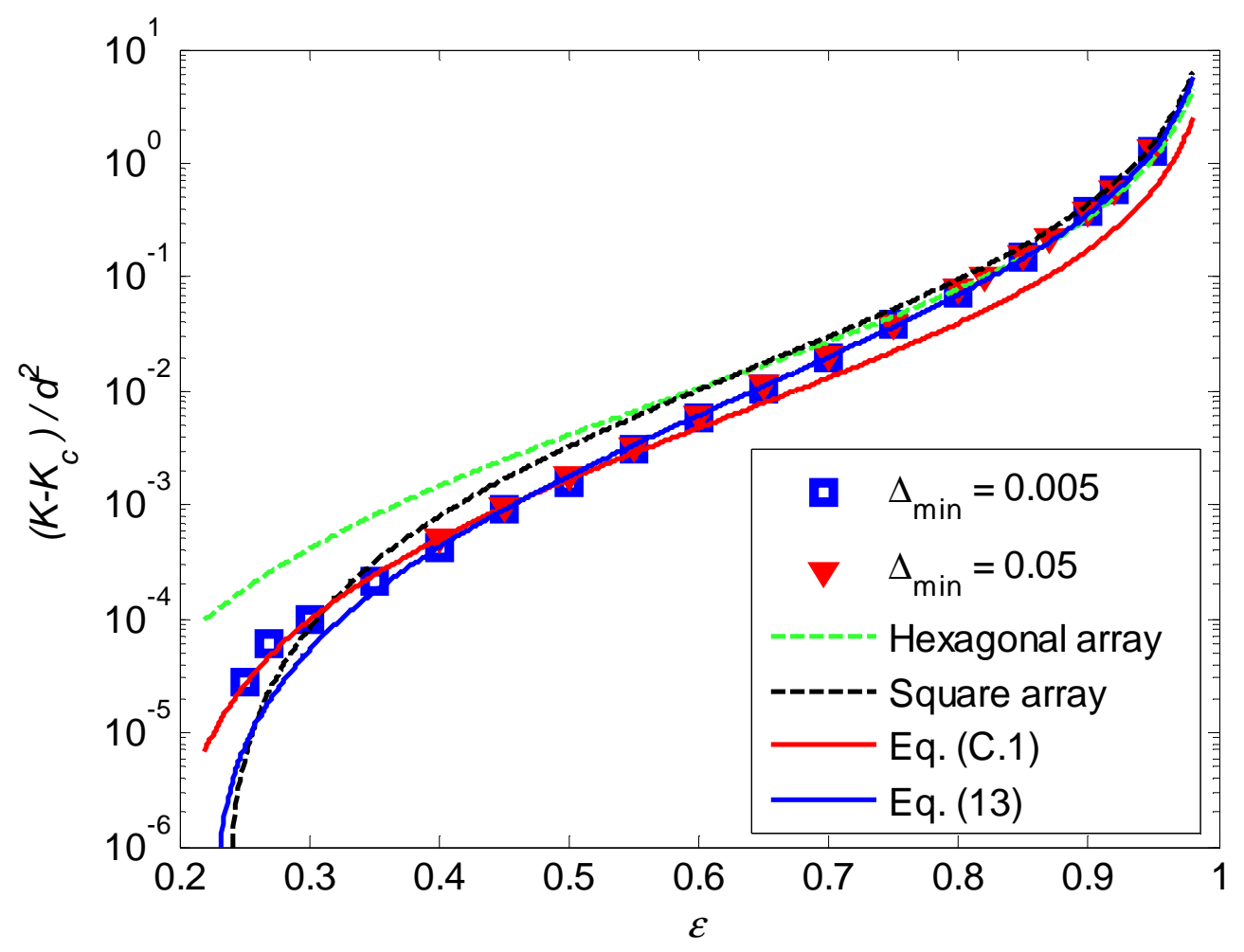

Figure C1. 